\title{
GW170104: Observation of a 50-Solar-Mass Binary Black Hole Coalescence at Redshift 0.2
}

\author{
B. P. Abbott et al. \\ (LIGO Scientific and Virgo Collaboration) \\ (Received 9 May 2017; published 1 June 2017)
}

\begin{abstract}
We describe the observation of GW170104, a gravitational-wave signal produced by the coalescence of a pair of stellar-mass black holes. The signal was measured on January 4, 2017 at 10:11:58.6 UTC by the twin advanced detectors of the Laser Interferometer Gravitational-Wave Observatory during their second observing run, with a network signal-to-noise ratio of 13 and a false alarm rate less than 1 in 70000 years. The inferred component black hole masses are $31.2_{-6.0}^{+8.4} M_{\odot}$ and $19.4_{-5.9}^{+5.3} M_{\odot}$ (at the $90 \%$ credible level). The black hole spins are best constrained through measurement of the effective inspiral spin parameter, a mass-weighted combination of the spin components perpendicular to the orbital plane, $\chi_{\text {eff }}=-0.12_{-0.30}^{+0.21}$. This result implies that spin configurations with both component spins positively aligned with the orbital angular momentum are disfavored. The source luminosity distance is $880_{-390}^{+450} \mathrm{Mpc}$ corresponding to a redshift of $z=0.18_{-0.07}^{+0.08}$. We constrain the magnitude of modifications to the gravitational-wave dispersion relation and perform null tests of general relativity. Assuming that gravitons are dispersed in vacuum like massive particles, we bound the graviton mass to $m_{g} \leq 7.7 \times 10^{-23} \mathrm{eV} / c^{2}$. In all cases, we find that GW170104 is consistent with general relativity.
\end{abstract}

DOI: 10.1103/PhysRevLett.118.221101

\section{INTRODUCTION}

The first observing run of the Advanced Laser Interferometer Gravitational-Wave Observatory (LIGO) [1] identified two binary black hole coalescence signals with high statistical significance, GW150914 [2] and GW151226 [3], as well as a less significant candidate LVT151012 [4,5]. These discoveries ushered in a new era of observational astronomy, allowing us to investigate the astrophysics of binary black holes and test general relativity (GR) in ways that were previously inaccessible [6,7]. We now know that there is a population of binary black holes with component masses $\gtrsim 25 M_{\odot}[5,6]$, and that merger rates are high enough for us to expect more detections $[5,8]$.

Advanced LIGO's second observing run began on November 30, 2016. On January 4, 2017, a gravitationalwave signal was detected with high statistical significance. Figure 1 shows a time-frequency representation of the data from the LIGO Hanford and Livingston detectors, with the signal GW170104 visible as the characteristic chirp of a binary coalescence. Detailed analyses demonstrate that GW170104 arrived at Hanford $\sim 3 \mathrm{~ms}$ before Livingston, and originated from the coalescence of two stellar-mass black holes at a luminosity distance of $\sim 3 \times 10^{9}$ light-years.

*Full author list given at the end of the Letter.

Published by the American Physical Society under the terms of the Creative Commons Attribution 4.0 International license. Further distribution of this work must maintain attribution to the author(s) and the published article's title, journal citation, and DOI.
GW170104's source is a heavy binary black hole system, with a total mass of $\sim 50 M_{\odot}$, suggesting formation in a subsolar metallicity environment [6]. Measurements of the black hole spins show a preference away from being (positively) aligned with the orbital angular momentum, but do not exclude zero spins. This is distinct from the case for GW151226, which had a strong preference for spins with positive projections along the orbital angular momentum [3]. The inferred merger rate agrees with previous calculations $[5,8]$, and could potentially be explained by binary black holes forming through isolated binary evolution or dynamical interactions in dense stellar clusters [6].

Gravitational-wave observations of binary black holes are the ideal means to test GR and its alternatives. They provide insight into regimes of strong-field gravity where velocities are relativistic and the spacetime is dynamic. The tests performed with the sources detected in the first observing run showed no evidence of departure from GR's predictions [5,7]; GW170104 provides an opportunity to tighten these constraints. In addition to repeating tests performed in the first observing run, we also test for modifications to the gravitational-wave dispersion relation. Combining measurements from GW170104 with our previous results, we obtain new gravitational-wave constraints on potential deviations from GR.

\section{DETECTORS AND DATA QUALITY}

The LIGO detectors measure gravitational-wave strain using two dual-recycled Fabry-Perot Michelson interferometers at the Hanford and Livingston observatories $[1,10]$. 


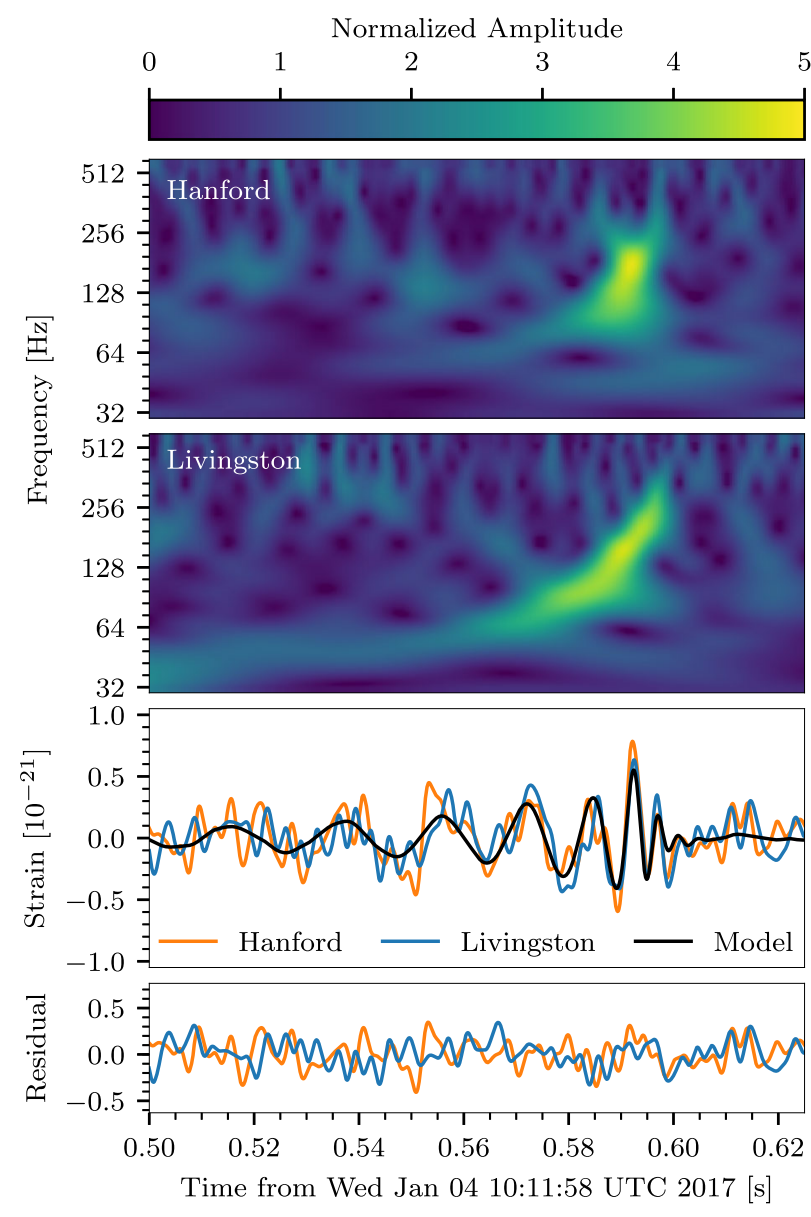

FIG. 1. Time-frequency representation [9] of strain data from Hanford and Livingston detectors (top two panels) at the time of GW170104. The data begin at 1167559936.5 GPS time. The third panel from the top shows the time-series data from each detector with a 30-350 Hz bandpass filter, and band-reject filters to suppress strong instrumental spectral lines. The Livingston data have been shifted back by $3 \mathrm{~ms}$ to account for the source's sky location, and the sign of its amplitude has been inverted to account for the detectors' different orientations. The maximumlikelihood binary black hole waveform given by the full-precession model (see Sec. IV) is shown in black. The bottom panel shows the residuals between each data stream and the maximumlikelihood waveform.

After the first observing run, both LIGO detectors underwent commissioning to reduce instrumental noise, and to improve duty factor and data quality (see Sec. I in the Supplemental Material [11]). For the Hanford detector, a high-power laser stage was introduced, and as the first step the laser power was increased from 22 to $30 \mathrm{~W}$ to reduce shot noise [10] at high frequencies. For the Livingston detector, the laser power was unchanged, but there was a significant improvement in low-frequency performance mainly due to the mitigation of scattered light noise.

Calibration of the interferometers is performed by inducing test-mass motion using photon pressure from modulated calibration lasers $[12,13]$. The one-sigma calibration uncertainties for strain data in both detectors for the times used in this analysis are better than 5\% in amplitude and $3^{\circ}$ in phase over the frequency range 20 $1024 \mathrm{~Hz}$.

At the time of GW170104, both LIGO detectors were operating with sensitivity typical of the observing run to date and were in an observation-ready state. Investigations similar to the detection validation procedures for previous events [2,14] found no evidence that instrumental or environmental disturbances contributed to GW170104.

\section{SEARCHES}

GW170104 was first identified by inspection of lowlatency triggers from Livingston data [15-17]. An automated notification was not generated as the Hanford detector's calibration state was temporarily set incorrectly in the low-latency system. After it was manually determined that the calibration of both detectors was in a nominal state, an alert with an initial source localization $[18,19]$ was distributed to collaborating astronomers [20] for the purpose of searching for a transient counterpart. About 30 groups of observers covered the parts of the sky localization using ground- and space-based instruments, spanning from $\gamma$ ray to radio frequencies as well as highenergy neutrinos [21].

Offline analyses are used to determine the significance of candidate events. They benefit from improved calibration and refined data quality information that is unavailable to low-latency analyses $[5,14]$. The second observing run is divided into periods of two-detector cumulative coincident observing time with $\gtrsim 5$ days of data to measure the false alarm rate of the search at the level where detections can be confidently claimed. Two independently designed matched filter analyses [16,22] used 5.5 days of coincident data collected from January 4, 2017 to January 22, 2017.

These analyses search for binary coalescences over a range of possible masses and by using discrete banks [23-28] of waveform templates modeling binaries with component spins aligned or antialigned with the orbital angular momentum [29]. The searches can target binary black hole mergers with detector-frame total masses $2 M_{\odot} \leq M^{\text {det }} \lesssim 100-500 M_{\odot}$, and spin magnitudes up to $\sim 0.99$. The upper mass boundary of the bank is determined by imposing a lower limit on the duration of the template in the detectors' sensitive frequency band [30]. Candidate events must be found in both detectors by the same template within $15 \mathrm{~ms}$ [4]. This 15-ms window is determined by the 10-ms intersite propagation time plus an allowance for the uncertainty in identified signal arrival times of weak signals. Candidate events are assigned a detection statistic value ranking their relative likelihood of being a gravitational-wave signal: the search uses an improved detection statistic compared to the first observing run [31]. The significance of a candidate event is calculated by comparing its detection statistic value to an estimate of the background noise $[4,16,17,22]$. GW170104 was detected 
with a network matched-filter signal-to-noise ratio (SNR) of 13. At the detection statistic value assigned to GW170104, the false alarm rate is less than 1 in 70000 years of coincident observing time.

The probability of astrophysical origin $P_{\text {astro }}$ for a candidate event is found by comparing the candidate's detection statistic to a model described by the distributions and rates of both background and signal events $[8,32,33]$. The background distribution is analysis dependent, being derived from the background samples used to calculate the false alarm rate. The signal distribution can depend on the mass distribution of the source systems; however, we find that different models of the binary black hole mass distribution (as described in Sec. VI) lead to negligible differences in the resulting value of $P_{\text {astro. }}$. At the detection statistic value of GW170104, the background rate in both matched filter analyses is dwarfed by the signal rate, yielding $P_{\text {astro }}>1-\left(3 \times 10^{-5}\right)$.

An independent analysis that is not based on matched filtering, but instead looks for generic gravitational-wave bursts $[2,34]$ and selects events where the signal frequency rises over time [35], also identified GW170104. This approach allows for signal deviations from the waveform models used for matched filtering at the cost of a lower significance for signals that are represented by the considered templates. This analysis reports a false alarm rate of $\sim 1$ in 20000 years for GW170104.

\section{SOURCE PROPERTIES}

The source parameters are inferred from a coherent Bayesian analysis of the data from both detectors [36,37]. As a cross-check, we use two independent model-waveform families. Both are tuned to numerical-relativity simulations of binary black holes with nonprecessing spins, and introduce precession effects through approximate prescriptions. One model includes inspiral spin precession using a single effective spin parameter $\chi_{p}$ [38-40]; the other includes the generic two-spin inspiral precession dynamics [41-43]. We refer to these as the effective-precession and full-precession models, respectively [44]. The two models yield consistent results. Table I shows selected source parameters for GW170104; unless otherwise noted, we quote the median and symmetric $90 \%$ credible interval for inferred quantities. The final mass (or equivalently the energy radiated), final spin, and peak luminosity are computed using averages of fits to numerical-relativity results [45-49]. The parameter uncertainties include statistical and systematic errors from averaging posterior probability distributions over the two waveform models, as well as calibration uncertainty [37] (and systematic uncertainty in the fit for peak luminosity). Statistical uncertainty dominates the overall uncertainty as a consequence of the moderate SNR.

For binary coalescences, the gravitational-wave frequency evolution is primarily determined by the component masses. For higher mass binaries, merger and ringdown dominate the
TABLE I. Source properties for GW170104: median values with $90 \%$ credible intervals. We quote source-frame masses; to convert to the detector frame, multiply by $(1+z)[50,51]$. The redshift assumes a flat cosmology with Hubble parameter $H_{0}=$ $67.9 \mathrm{~km} \mathrm{~s}^{-1} \mathrm{Mpc}^{-1}$ and matter density parameter $\Omega_{m}=0.3065$ [52]. More source properties are given in Table I of the Supplemental Material [11].

\begin{tabular}{lc}
\hline \hline Primary black hole mass $m_{1}$ & $31.2_{-6.0}^{+8.4} M_{\odot}$ \\
Secondary black hole mass $m_{2}$ & $19.4_{-5.9}^{+5.3} M_{\odot}$ \\
Chirp mass $\mathcal{M}$ & $21.1_{-2.7}^{+2.4} M_{\odot}$ \\
Total mass $M$ & $50.7_{-5.0}^{+5.9} M_{\odot}$ \\
Final black hole mass $M_{f}$ & $48.7_{-4.6}^{+5.7} M_{\odot}$ \\
Radiated energy $E_{\text {rad }}$ & $2.0_{-0.7}^{+0.6} M_{\odot} c^{2}$ \\
Peak luminosity $\ell_{\text {peak }}$ & $3.1_{-1.3}^{+0.7} \times 10^{56} \mathrm{erg} \mathrm{s}^{-1}$ \\
Effective inspiral spin parameter $\chi_{\text {eff }}$ & $-0.12_{-0.30}^{+0.21}$ \\
Final black hole spin $a_{f}$ & $0.64_{-0.20}^{+0.09}$ \\
Luminosity distance $D_{L}$ & $880_{-390}^{+450} \mathrm{Mpc}$ \\
Source redshift $z$ & $0.18_{-0.07}^{+0.08}$ \\
\hline \hline
\end{tabular}

signal, allowing good measurements of the total mass $M=$ $m_{1}+m_{2}$ [53-57]. For lower mass binaries, like GW151226 [3], the inspiral is more important, providing precision measurements of the chirp mass $\mathcal{M}=\left(m_{1} m_{2}\right)^{3 / 5} / M^{1 / 5}$ [58-61]. The transition between the regimes depends upon the detectors' sensitivity, and GW170104 sits between the

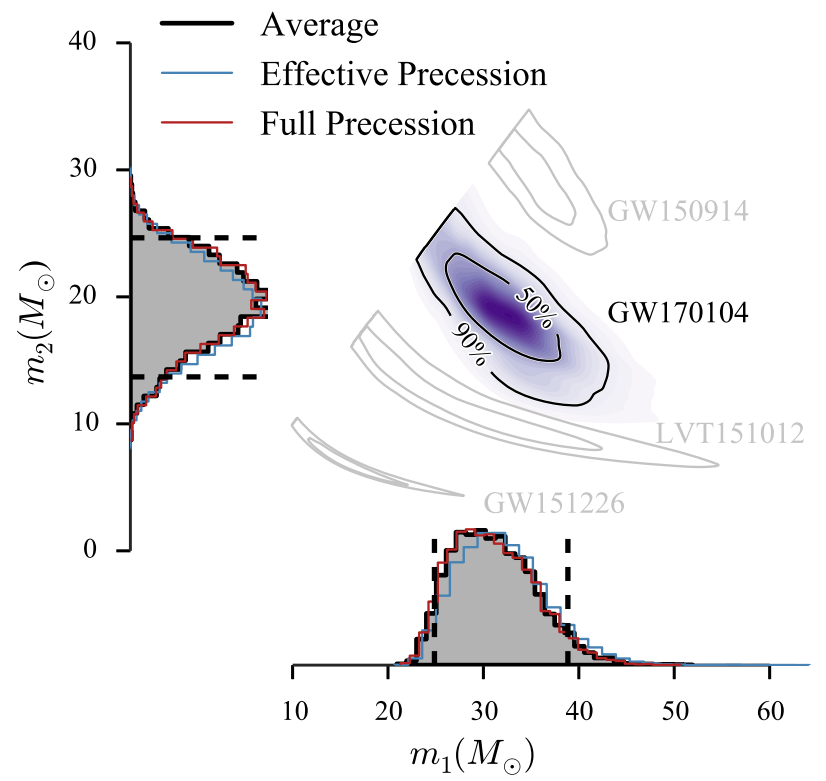

FIG. 2. Posterior probability density for the source-frame masses $m_{1}$ and $m_{2}$ (with $m_{1} \geq m_{2}$ ). The one-dimensional distributions include the posteriors for the two waveform models, and their average (black). The dashed lines mark the $90 \%$ credible interval for the average posterior. The two-dimensional plot shows the contours of the $50 \%$ and $90 \%$ credible regions plotted over a colorcoded posterior density function. For comparison, we also show the two-dimensional contours for the previous events [5]. 
two. The inferred component masses are shown in Fig. 2. The form of the two-dimensional distribution is guided by the combination of constraints on $M$ and $\mathcal{M}$. The binary was composed of two black holes with masses $m_{1}=31.2_{-6.0}^{+8.4} M_{\odot}$ and $m_{2}=19.4_{-5.9}^{+5.3} M_{\odot}$; these merged into a final black hole of mass $48.7_{-4.6}^{+5.7} M_{\odot}$. This binary ranks second, behind GW150914's source [5,37], as the most massive stellar-mass binary black hole system observed to date.

The black hole spins play a subdominant role in the orbital evolution of the binary, and are more difficult to determine. The orientations of the spins evolve due to precession $[62,63]$, and we report results at a point in the inspiral corresponding to a gravitational-wave frequency of $20 \mathrm{~Hz}$ [37]. The effective inspiral spin parameter $\chi_{\text {eff }}=$ $\left(m_{1} a_{1} \cos \theta_{L S_{1}}+m_{2} a_{2} \cos \theta_{L S_{2}}\right) / M$ is the most important spin combination for setting the properties of the inspiral [64-66] and remains important through to merger [67-71]; it is approximately constant throughout the orbital evolution [72,73]. Here $\theta_{L S_{i}}=\cos ^{-1}\left(\hat{\mathbf{L}} \cdot \hat{\mathbf{S}}_{i}\right)$ is the tilt angle between the spin $\mathbf{S}_{i}$ and the orbital angular momentum $\mathbf{L}$, which ranges from $0^{\circ}$ (spin aligned with orbital angular momentum) to $180^{\circ}$ (spin antialigned); $a_{i}=\left|c \mathbf{S}_{i} / G m_{i}^{2}\right|$ is the (dimensionless) spin magnitude, which ranges from 0 to 1 , and $i=1$ for the primary black hole and $i=2$ for the secondary. We use the Newtonian angular momentum for $\mathbf{L}$, such that it is normal to the orbital plane; the total orbital angular momentum differs from this because of postNewtonian corrections. We infer that $\chi_{\text {eff }}=-0.12_{-0.30}^{+0.21}$. Similarly to GW150914 $[5,37,44], \chi_{\text {eff }}$ is close to zero with a preference towards being negative: the probability that $\chi_{\text {eff }}<0$ is 0.82 . Our measurements therefore disfavor a large total spin positively aligned with the orbital angular momentum, but do not exclude zero spins.

The in-plane components of the spin control the amount of precession of the orbit [62]. This may be quantified by the effective precession spin parameter $\chi_{p}$ which ranges from 0 (no precession) to 1 (maximal precession) [39]. Figure 3 (top) shows the posterior probability density for $\chi_{\text {eff }}$ and $\chi_{p}$ [39]. We gain some information on $\chi_{\text {eff }}$, excluding large positive values, but, as for previous events $[3,5,37]$, the $\chi_{p}$ posterior is dominated by the prior (see Sec. III of the Supplemental Material [11]). No meaningful constraints can be placed on the magnitudes of the in-plane spin components and hence precession.

The inferred component spin magnitudes and orientations are shown in Fig. 3 (bottom). The lack of constraints on the in-plane spin components means that we learn almost nothing about the spin magnitudes. The secondary's spin is less well constrained as the less massive component has a smaller impact on the signal. The probability that the tilt $\theta_{L S_{i}}$ is less than $45^{\circ}$ is 0.04 for the primary black hole and 0.08 for the secondary, whereas the prior probability is 0.15 for each. Considering the two spins together, the probability that both tilt angles are less than $90^{\circ}$ is 0.05 .
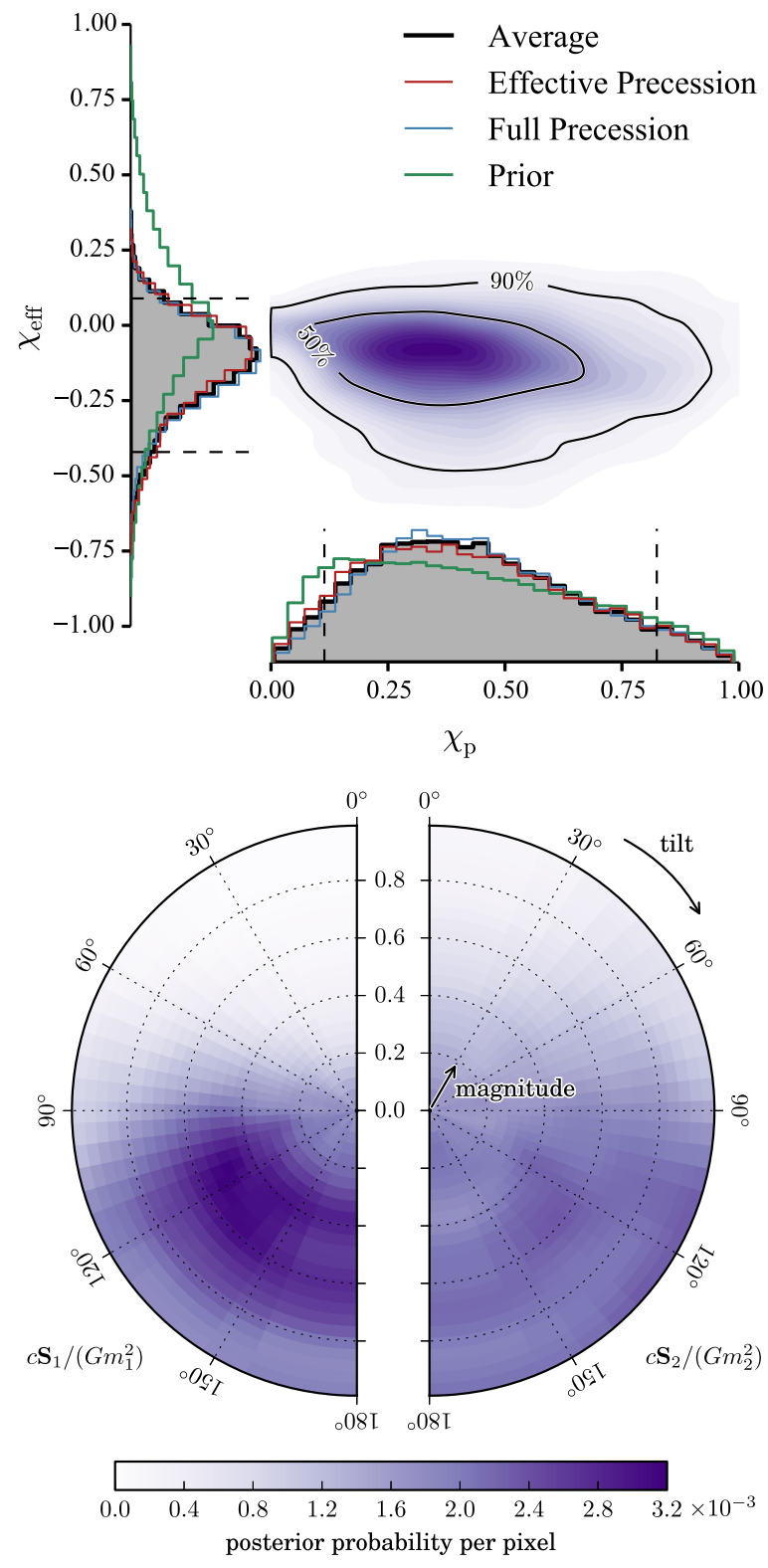

FIG. 3. Top: Posterior probability density for the effective inspiral and precession spin parameters, $\chi_{\text {eff }}$ and $\chi_{p}$. The one-dimensional distributions show the posteriors for the two waveform models, their average (black), and the prior distributions (green). The dashed lines mark the $90 \%$ credible interval for the average posterior. The two-dimensional plot shows the $50 \%$ and $90 \%$ credible regions plotted over the posterior density function. Bottom: Posterior probabilities for the dimensionless component spins, $c \mathbf{S}_{1} /\left(G m_{1}^{2}\right)$ and $c \mathbf{S}_{2} /\left(G m_{2}^{2}\right)$, relative to the normal of the orbital plane $\hat{\mathbf{L}}$. The tilt angles are $0^{\circ}$ for spins aligned with the orbital angular momentum and $180^{\circ}$ for spins antialigned. The probabilities are marginalized over the azimuthal angles. The pixels have equal prior probability $\left(1.6 \times 10^{-3}\right)$; they are spaced linearly in spin magnitudes and the cosine of the tilt angles. Results are given at a gravitational-wave frequency of $20 \mathrm{~Hz}$. 
Effectively all of the information comes from constraints on $\chi_{\text {eff }}$ combined with the mass ratio (and our prior of isotropically distributed orientations and uniformly distributed magnitudes) [5].

The source's luminosity distance $D_{L}$ is inferred from the signal amplitude $[37,74]$. The amplitude is inversely proportional to the distance, but also depends upon the binary's inclination [59,75-77]. This degeneracy is a significant source of uncertainty $[57,71]$. The inclination has a bimodal distribution with broad peaks for face-on and face-off orientations (see Fig. 4 of the Supplemental Material [11]). GW170104's source is at $D_{L}=880_{-390}^{+450} \mathrm{Mpc}$, corresponding to a cosmological redshift of $z=0.18_{-0.07}^{+0.08}$ [52]. While GW170104's source has masses and spins comparable to GW150914's, it is most probably at a greater distance [5,37].

For GW150914, extensive studies were made to verify the accuracy of the model waveforms for parameter estimation through comparisons with numerical-relativity waveforms [78,79]. GW170104 is a similar system to GW150914 and, therefore, it is unlikely that there are any significant biases in our results as a consequence of waveform modeling. The lower SNR of GW170104 makes additional effects not incorporated in the waveform models, such as higher modes $[55,80,81]$, less important. However, if the source is edge on or strongly precessing, there could be significant biases in quantities including $\mathcal{M}$ and $\chi_{\text {eff }}$ [78]. Comparison to numerical-relativity simulations of binary black holes with nonprecessing spins [79], including those designed to replicate GW170104, produced results (and residuals) consistent with the model-waveform analysis.

\section{WAVEFORM RECONSTRUCTIONS}

Consistency of GW170104 with binary black hole waveform models can also be explored through comparisons with a morphology-independent signal model [82]. We choose to describe the signal as a superposition of an arbitrary number of Morlet-Gabor wavelets, which models an elliptically polarized, coherent signal in the detector network. Figure 4 plots whitened detector data at the time of GW170104, together with waveforms drawn from the $90 \%$ credible region of the posterior distributions of the morphology-independent model and the binary black hole waveform models used to infer the source properties. The signal appears in the two detectors with slightly different amplitudes, and a relative phase shift of approximately $180^{\circ}$, because of their different spatial orientations [2]. The wavelet- and template-based reconstructions differ at early times because the wavelet basis requires high-amplitude, welllocalized signal energy to justify the presence of additional wavelets, while the earlier portion of the signal is inherently included in the binary black hole waveform model.

The waveforms reconstructed from the morphologyindependent model are consistent with the characteristic inspiral-merger-ringdown structure. The overlap [58]

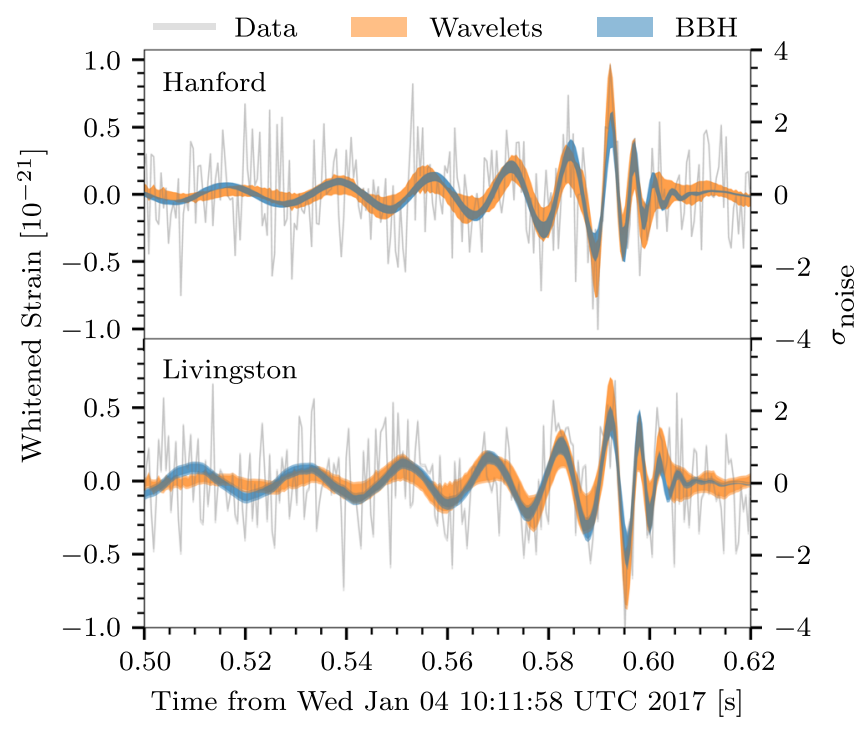

FIG. 4. Time-domain detector data (gray), and $90 \%$ confidence intervals for waveforms reconstructed from the morphologyindependent wavelet analysis (orange) and binary black hole $(\mathrm{BBH})$ models from both waveform families (blue), whitened by each instrument's noise amplitude spectral density. The left ordinate axes are normalized such that the amplitude of the whitened data and the physical strain are equal at $200 \mathrm{~Hz}$. The right ordinate axes are in units of noise standard deviations. The width of the $\mathrm{BBH}$ region is dominated by the uncertainty in the astrophysical parameters.

between the maximum-likelihood waveform of the binary black hole model and the median waveform of the morphology-independent analysis is $87 \%$, consistent with expectations from Monte Carlo analysis of binary black hole signals injected into detector data [34]. We also use the morphologyindependent analysis to search for residual gravitationalwave energy after subtracting the maximum-likelihood binary black hole signal from the measured strain data. There is an $83 \%$ posterior probability in favor of Gaussian noise versus residual coherent gravitational-wave energy which is not described by the waveform model, implying that GW170104's source is a black hole binary.

\section{BINARY BLACK HOLE POPULATIONS AND MERGER RATES}

The addition of the first 11 days of coincident observing time in the second observing run, and the detection of GW170104, leads to an improved estimate of the rate density of binary black hole mergers. We adopt two simple representative astrophysical population models: a distribution that is a power law in $m_{1}$ and uniform in $m_{2}$, $p\left(m_{1}, m_{2}\right) \propto m_{1}^{-\alpha} /\left(m_{1}-5 M_{\odot}\right)$ with $\alpha=2.35$ [83], and a distribution uniform in the logarithm of each of the component masses [5,8]. In both cases, we impose $m_{1}, m_{2} \geq 5 M_{\odot}$ and $M \leq 100 M_{\odot}$ [8]. Using the results from the first observing run as a prior, we obtain updated rates estimates of $R=103_{-63}^{+110} \mathrm{Gpc}^{-3} \mathrm{yr}^{-1}$ for the power 
law, and $R=32_{-20}^{+33} \mathrm{Gpc}^{-3} \mathrm{yr}^{-1}$ for the uniform-in-log distribution [5]. These combine search results from the two offline matched filter analyses, and marginalize over the calibration uncertainty [32]. The range for the merger rate that brackets the two distributions, $12-213 \mathrm{Gpc}^{-3} \mathrm{yr}^{-1}$, is consistent with the range $9-240 \mathrm{Gpc}^{-3} \mathrm{yr}^{-1}$ estimated from the first observing run [5,8]. Recalculating the rates directly after observing a new event can bias rate estimates, but this bias decreases with increasing event count and is negligible compared to other uncertainties on the intervals. While the median estimates have not changed appreciably, the overall tightening in the credible intervals is consistent with the additional observation time and the increment in the number of events with significant probability of being astrophysical from 3 to 4 .

Following the first observing run, we performed a hierarchical analysis using the inferred masses of GW150914, LVT151012, and GW151226 to constrain the binary black hole mass distribution. We assumed the power-law population distribution described above, treating $\alpha$ as a parameter to be estimated, and found $\alpha=2.5_{-1.6}^{+1.5}$ [5]. With the addition of GW170104, $\alpha$ is estimated to be $2.3_{-1.4}^{+1.3}$ (see Sec. IV of the Supplemental Material [11]); the median is close to the power-law exponent used to infer the (higher) merger rates.

\section{ASTROPHYSICAL IMPLICATIONS}

GW170104's source is a heavy stellar-mass binary black hole system. Such binaries are consistent with formation through several different evolutionary pathways [6]. Assuming black holes of stellar origin, there are two broad families of formation channels: dynamical and isolated binary evolution. Dynamical assembly of binaries is expected in dense stellar clusters [84-91]. Dynamical influences are also important for binary coalescences near galactic nuclei [92-94], and through interactions as part of a triple $[95,96]$. Isolated binary evolution in galactic fields classically proceeds via a common envelope [97-105]. Variants avoiding common-envelope evolution include (quasi-)chemically homogeneous evolution of massive tidally locked binaries $[101,106,107]$, or through stable mass transfer in Population I $[108,109]$ or Population III binaries [110,111].

Stars lose mass throughout their lives; to leave a heavy black hole as a remnant they must avoid significant mass loss. Low-metallicity progenitors are believed to have weaker stellar winds and hence diminished mass loss [112]. Given the mass of the primary black hole, the progenitors of GW170104 likely formed in a lower metallicity environment $Z \lesssim 0.5 Z_{\odot}[6,100,113-115]$, but low mass loss may also have been possible at higher metallicity if the stars were strongly magnetized [116].

An alternative to the stellar-evolution channels would be binaries of primordial black holes [117-120]. GW170104's component masses lie in a range for which primordial black holes could contribute significantly to the dark matter content of the Universe, but merger rates in such scenarios are uncertain $[118,121]$. The potential for existing electromagnetic observations to exclude primordial black holes of these masses is an active area of research [119,122-128].

Some of the formation models listed above predict merger rates on the order of $\sim 1-10 \mathrm{Gpc}^{-3} \mathrm{yr}^{-1} \quad[85,87,92-$ $96,107,110,115]$. Given that the rate intervals have now tightened and the lower bound (from the uniform-in-log distribution) is $\sim 12 \mathrm{Gpc}^{-3} \mathrm{yr}^{-1}$, these channels may be insufficient to explain the full rate, but they could contribute to the total rate if there are multiple channels in operation. Future observations will improve the precision of the rate estimation, its redshift dependence, and our knowledge of the mass distribution, making it easier to constrain binary formation channels.

Gravitational-wave observations provide information about the component spins through measurements of $\chi_{\text {eff }}$, and these measurements can potentially be used to distinguish different formation channels. Dynamically assembled binaries (of both stellar and primordial black holes) should have an isotropic distribution of spin tilts, with equal probability for positive and negative $\chi_{\text {eff }}$, and a concentration around zero [129]. Isolated binary evolution typically predicts moderate $\left(\lesssim 45^{\circ}\right)$ spin misalignments [130], since the effect of many astrophysical processes, such as mass transfer $[131,132]$ and tides [133,134], is to align spins with the orbital angular momentum. Black hole spins could become misaligned due to supernova explosions or torques during collapse. Large natal kicks are needed to produce negative $\chi_{\text {eff }}$ by changing the orbital plane $[129,130,135]$. The magnitude of these kicks is currently uncertain [136-141] and also influences the merger rate, with high kicks producing lower merger rates in some population-synthesis models $[98,100,115,142]$. For binary neutron stars there is evidence that large tilts may be possible with small kicks [143-146], and it is not yet understood if similar torques could occur for black holes [138,147-149]. The absolute value of $\chi_{\text {eff }}$ depends on the spin magnitudes. Small values of $\left|\chi_{\text {eff }}\right|$ can arise because the spin magnitudes are low, or because they are misaligned with the orbital angular momentum or each other. The spin magnitudes for binary black holes are currently uncertain, but GW151226 demonstrated that they can be $\gtrsim 0.2$ [3], and high-mass $x$-ray binary measurements indicate that the distribution of black hole spins could extend to larger magnitudes [147]. For GW170104, we infer $\chi_{\text {eff }}=-0.12_{-0.30}^{+0.21}$. This includes the possibility of negative $\chi_{\text {eff }}$, which would indicate spin-orbit misalignment of at least one component. It also excludes large positive values, and thus could argue against its source forming through chemically homogeneous evolution, since large aligned spins $\left(a_{i} \gtrsim 0.4\right)$ would be expected assuming the complete collapse of the progenitor stars [106]. The inferred range is consistent with dynamical assembly and isolated binary 
evolution provided that the positive orbit-aligned spin is small (whether due to low spins or misalignment) $[129,150$ 152]. Current gravitational-wave measurements cluster around $\chi_{\text {eff }} \sim 0\left(\left|\chi_{\text {eff }}\right|<0.35\right.$ at the $90 \%$ credible level for all events; see Fig. 5 of the Supplemental Material [11]) [5]. Assuming that binary black hole spins are not typically small $(\lesssim 0.2)$, our observations hint towards the astrophysical population favoring a distribution of misaligned spins rather than near orbit-aligned spins [153]; further detections will test if this is the case, and enable us to distinguish different spin magnitude and orientation distributions [154-159].

\section{TESTS OF GENERAL RELATIVITY}

To check the consistency of the observed signals with the predictions of GR for binary black holes in quasicircular orbit, we employ a phenomenological approach that probes how gravitational-wave generation or propagation could be modified in an alternative theory of gravity. Testing for these characteristic modifications in the waveform can quantify the degree to which departures from GR can be tolerated given the data. First, we consider the possibility of a modified gravitational-wave dispersion relation, and place bounds on the magnitude of potential deviations from GR. Second, we perform null tests to quantify generic deviations from GR: without assuming a specific alternative theory of gravity, we verify if the detected signal is compatible with GR. For these tests we use the three confident detections (GW150914, GW151226, and GW170104); we do not use the marginal event LVT151012, as its low SNR means that it contributes insignificantly to all the tests [5].

\section{A. Modified dispersion}

In GR, gravitational waves are nondispersive. We consider a modified dispersion relation of the form $E^{2}=$ $p^{2} c^{2}+A p^{\alpha} c^{\alpha}, \alpha \geq 0$, that leads to dephasing of the waves relative to the phase evolution in GR. Here $E$ and $p$ are the energy and momentum of gravitational radiation, and $A$ is the amplitude of the dispersion [160,161]. Modifications to the dispersion relation can arise in theories that include violations of local Lorentz invariance [162]. Lorentz invariance is a cornerstone of modern physics but its violation is expected in certain quantum gravity frameworks $[162,163]$. Several modified theories of gravity predict specific values of $\alpha$, including massive-graviton theories $(\alpha=0, A>0)$ [163], multifractal spacetime [164] $(\alpha=2.5)$, doubly special relativity [165] $(\alpha=3)$, and Hořava-Lifshitz [166] and extradimensional [167] theories $(\alpha=4)$. For our analysis, we assume that the only effect of these alternative theories is to modify the dispersion relation.

To leading order in $A E^{\alpha-2}$, the group velocity of gravitational waves is modified as $v_{g} / c=1+(\alpha-1) A E^{\alpha-2} / 2$ [161]; both superluminal and subluminal propagation velocities are possible, depending on the sign of $A$ and the value of $\alpha$. A change in the dispersion relation leads to an extra term

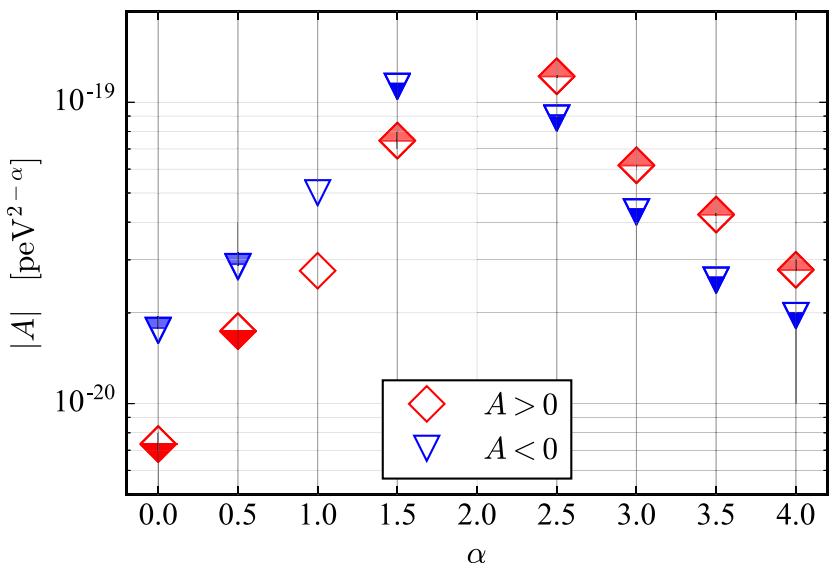

FIG. 5. $90 \%$ credible upper bounds on $|A|$, the magnitude of dispersion, obtained combining the posteriors of GW170104 with those of GW150914 and GW151226. We use picoelectronvolts as a convenient unit because the corresponding frequency scale is around where GW170104 has greatest amplitude ( $1 \mathrm{peV} \simeq h \times 250 \mathrm{~Hz}$, where $h$ is the Planck constant). General relativity corresponds to $A=0$. Markers filled at the top (bottom) correspond to values of $|A|$ and $\alpha$ for which gravitational waves travel with superluminal (subluminal) speed.

$\delta \Psi(A, \alpha)$ in the evolution of the gravitational-wave phase [160]. We introduce such a term in the effective-precession waveform model [38] to constrain dispersion for various values of $\alpha$. To this end, we assume flat priors on $A$. In Fig. 5 we show $90 \%$ credible upper bounds on $|A|$ derived from the three confident detections. We do not show results for $\alpha=2$ since in this case the modification of the gravitational-wave phase is degenerate with the arrival time of the signal.

There exist constraints on Lorentz invariance violating dispersion relations from other observational sectors (e.g., photon or neutrino observations) for certain values of $\alpha$, and our results are weaker by several orders of magnitude. However, there are frameworks in which Lorentz invariance is only broken in one sector [168,169], implying that each sector provides complementary information on potential modifications to GR. Our results are the first bounds derived from gravitational-wave observations, and the first tests of superluminal propagation in the gravitational sector.

The result for $A>0$ and $\alpha=0$ can be reparametrized to derive a lower bound on the graviton Compton wavelength $\lambda_{g}$, assuming that gravitons disperse in vacuum in the same way as massive particles $[5,7,170]$. In this case, no violation of Lorentz invariance is assumed. Using a flat prior for the graviton mass, we obtain $\lambda_{g}>1.5 \times 10^{13} \mathrm{~km}$, which improves on the bound of $1.0 \times 10^{13} \mathrm{~km}$ from previous gravitationalwave observations [5,7]. The combined bound using the three confident detections is $\lambda_{g}>1.6 \times 10^{13} \mathrm{~km}$, or for the graviton mass $m_{g} \leq 7.7 \times 10^{-23} \mathrm{eV} / c^{2}$.

\section{B. Null tests}

In the post-Newtonian approximation, the gravitationalwave phase in the Fourier domain is a series expansion in 
powers of frequency, the expansion coefficients being functions of the source parameters $[60,63,171]$. In the effective-precession model, waveforms from numericalrelativity simulations are also modeled using an expansion of the phase in terms of the Fourier frequency. To verify if the detected signal is consistent with GR, we allow the expansion coefficients to deviate in turn from their nominal GR value and we obtain a posterior distribution for the difference between the measured and GR values [172177]. We find no significant deviation from the predictions of GR [5,7]. Combined bounds for GW170104 and the two confident detections from the first observing run [5] do not significantly improve the bounds on the waveform phase coefficients.

Finally, we investigate whether the merger-ringdown portion of the detected signal is consistent with the inspiral part $[7,178,179]$. The two parts are divided at $143 \mathrm{~Hz}$, a frequency close to the median inferred (detector-frame) innermost-stable-circular-orbit frequency of the remnant Kerr black hole. For each part, we infer the component masses and spins, and calculate from these the final mass and spin using fits from numerical relativity, as in Sec. IV [4548]. We then calculate a two-dimensional posterior distribution for the fractional difference between final mass and spin calculated separately from the two parts $[7,179]$. The expected GR value (no difference in the final mass and spin estimates) lies close to the peak of the posterior distribution, well within the $90 \%$ credible region. When combined with the posteriors from GW150914, the width of the credible intervals decreases by a factor of $\sim 1.5$, providing a better constraint on potential deviations from GR.

In conclusion, in agreement with the predictions of GR, none of the tests we performed indicate a statistically significant departure from the coalescence of Kerr black holes in a quasicircular orbit.

\section{CONCLUSIONS}

Advanced LIGO began its second observing run on November 30, 2016, and on January 4, 2017 the LIGOHanford and LIGO-Livingston detectors registered a highly significant gravitational-wave signal GW170104 from the coalescence of two stellar-mass black holes. GW170104 joins two other high-significance events [2,3] and a marginal candidate [4] from Advanced LIGO's first observing run [5]. This new detection is entirely consistent with the astrophysical rates inferred from the previous run. The source is a heavy binary black hole system, similar to that of GW150914. Spin configurations with both component spins aligned with the orbital angular momentum are disfavored (but not excluded); we do not significantly constrain the component black holes' spin magnitudes. The observing run will continue until mid 2017. Expanding the catalog of binary black holes will provide further insight into their formation and evolution, and allow for tighter constraints on potential modifications to GR.
Further details of the analysis and the results are given in the Supplemental Material [11]. Data for this event are available at the LIGO Open Science Center [180].

\section{ACKNOWLEDGMENTS}

The authors gratefully acknowledge the support of the United States National Science Foundation (NSF) for the construction and operation of the LIGO Laboratory and Advanced LIGO as well as the Science and Technology Facilities Council (STFC) of the United Kingdom, the MaxPlanck-Society (MPS), and the State of Niedersachsen/ Germany for support of the construction of Advanced LIGO and construction and operation of the GEO 600 detector. Additional support for Advanced LIGO was provided by the Australian Research Council. The authors gratefully acknowledge the Italian Istituto Nazionale di Fisica Nucleare (INFN), the French Centre National de la Recherche Scientifique (CNRS) and the Foundation for Fundamental Research on Matter supported by the Netherlands Organisation for Scientific Research, for the construction and operation of the Virgo detector and the creation and support of the EGO consortium. The authors also gratefully acknowledge research support from these agencies as well as by the Council of Scientific and Industrial Research of India, Department of Science and Technology, India, Science \& Engineering Research Board (SERB), India, Ministry of Human Resource Development, India, the Spanish Ministerio de Economía y Competitividad, the Vicepresidència i Conselleria d'Innovació, Recerca i Turisme and the Conselleria d'Educació i Universitat del Govern de les Illes Balears, the National Science Centre of Poland, the European Commission, the Royal Society, the Scottish Funding Council, the Scottish Universities Physics Alliance, the Hungarian Scientific Research Fund (OTKA), the Lyon Institute of Origins (LIO), the National Research Foundation of Korea, Industry Canada and the Province of Ontario through the Ministry of Economic Development and Innovation, the Natural Science and Engineering Research Council Canada, Canadian Institute for Advanced Research, the Brazilian Ministry of Science, Technology, and Innovation, International Center for Theoretical Physics South American Institute for Fundamental Research (ICTP-SAIFR), Russian Foundation for Basic Research, the Leverhulme Trust, the Research Corporation, Ministry of Science and Technology (MOST), Taiwan, and the Kavli Foundation. The authors gratefully acknowledge the support of the NSF, STFC, MPS, INFN, CNRS, and the State of Niedersachsen/Germany for provision of computational resources. This article has been assigned the document number LIGO-P170104.

[1] J. Aasi et al. (LIGO Scientific Collaboration), Classical Quantum Gravity 32, 074001 (2015). 
[2] B. P. Abbott et al. (LIGO Scientific Collaboration and Virgo Collaboration), Phys. Rev. Lett. 116, 061102 (2016).

[3] B. Abbott et al. (LIGO Scientific Collaboration and Virgo Collaboration), Phys. Rev. Lett. 116, 241103 (2016).

[4] B. P. Abbott et al. (LIGO Scientific Collaboration and Virgo Collaboration), Phys. Rev. D 93, 122003 (2016).

[5] B. P. Abbott et al. (LIGO Scientific Collaboration and Virgo Collaboration), Phys. Rev. X 6, 041015 (2016).

[6] B. P. Abbott et al. (LIGO Scientific Collaboration and Virgo Collaboration), Astrophys. J. Lett. 818, L22 (2016).

[7] B. P. Abbott et al. (LIGO Scientific Collaboration and Virgo Collaboration), Phys. Rev. Lett. 116, 221101 (2016).

[8] B. P. Abbott et al. (LIGO Scientific Collaboration and Virgo Collaboration), Astrophys. J. Lett. 833, L1 (2016).

[9] S. Chatterji, L. Blackburn, G. Martin, and E. Katsavounidis, Classical Quantum Gravity 21, S1809 (2004).

[10] B. P. Abbott et al. (LIGO Scientific Collaboration and Virgo Collaboration), Phys. Rev. Lett. 116, 131103 (2016).

[11] See Supplemental Material at http://link.aps.org/ supplemental/10.1103/PhysRevLett.118.221101 for technical details and additional results.

[12] S. Karki et al., Rev. Sci. Instrum. 87, 114503 (2016).

[13] B. P. Abbott et al. (LIGO Scientific Collaboration), Phys. Rev. D 95, 062003 (2017).

[14] B. P. Abbott et al. (LIGO Scientific Collaboration and Virgo Collaboration), Classical Quantum Gravity 33, 134001 (2016).

[15] T. Dal Canton et al., Phys. Rev. D 90, 082004 (2014).

[16] S. A. Usman et al., Classical Quantum Gravity 33, 215004 (2016).

[17] A. H. Nitz, I. W. Harry, J. L. Willis, C. M. Biwer, D. A. Brown, L. P. Pekowsky, T. Dal Canton, A. R. Williamson, T. Dent, C. D. Capano, T. J. Massinger, A. K. Lenon, A. B. Nielsen, and M. Cabero, PyCBC Software, https://github .com/ligo-cbc/pycbc (2017).

[18] L. P. Singer and L. R. Price, Phys. Rev. D 93, 024013 (2016).

[19] L. P. Singer et al., Astrophys. J. Lett. 829, L15 (2016).

[20] LIGO Scientific Collaboration and Virgo Collaboration, Gamma-ray Coordinates Network/Transient Astronomy Network Circular 20364 (2017) [https://gcn.gsfc.nasa .gov/gen3/20364.gen3].

[21] The collection of Circulars exchanged with collaborating astronomers regarding this event is archived at https://gcn .gsfc.nasa.gov/other/G268556.gcn3.

[22] C. Messick et al., Phys. Rev. D 95, 042001 (2017).

[23] C. Capano, I. Harry, S. Privitera, and A. Buonanno, Phys. Rev. D 93, 124007 (2016).

[24] D. A. Brown, I. Harry, A. Lundgren, and A. H. Nitz, Phys. Rev. D 86, 084017 (2012).

[25] I. W. Harry, B. Allen, and B. S. Sathyaprakash, Phys. Rev. D 80, 104014 (2009).

[26] P. Ajith, N. Fotopoulos, S. Privitera, A. Neunzert, N. Mazumder, and A. J. Weinstein, Phys. Rev. D 89, 084041 (2014).

[27] B. S. Sathyaprakash and S. V. Dhurandhar, Phys. Rev. D 44, 3819 (1991).

[28] B. J. Owen and B. S. Sathyaprakash, Phys. Rev. D 60, 022002 (1999).

[29] A. Bohé et al., Phys. Rev. D 95, 044028 (2017).
[30] T. Dal Canton and I. Harry, arXiv:1705.01845.

[31] A. H. Nitz, T. Dent, T. Dal Canton, S. Fairhurst, and D. Brown, arXiv:1705.01513.

[32] B. P. Abbott et al. (LIGO Scientific Collaboration and Virgo Collaboration), Astrophys. J. Suppl. Ser. 227, 14 (2016).

[33] W. M. Farr, J. R. Gair, I. Mandel, and C. Cutler, Phys. Rev. D 91, 023005 (2015).

[34] B. P. Abbott et al. (LIGO Scientific Collaboration and Virgo Collaboration), Phys. Rev. D 93, 122004 (2016); 94, 069903 (2016) (Addendum).

[35] S. Klimenko et al., Phys. Rev. D 93, 042004 (2016).

[36] J. Veitch et al., Phys. Rev. D 91, 042003 (2015).

[37] B. P. Abbott et al. (LIGO Scientific Collaboration and Virgo Collaboration), Phys. Rev. Lett. 116, 241102 (2016).

[38] M. Hannam, P. Schmidt, A. Bohé, L. Haegel, S. Husa, F. Ohme, G. Pratten, and M. Pürrer, Phys. Rev. Lett. 113, 151101 (2014).

[39] P. Schmidt, F. Ohme, and M. Hannam, Phys. Rev. D 91, 024043 (2015).

[40] S. Khan, S. Husa, M. Hannam, F. Ohme, M. Pürrer, X. Jiménez Forteza, and A. Bohé, Phys. Rev. D 93, 044007 (2016).

[41] Y. Pan, A. Buonanno, A. Taracchini, L. E. Kidder, A. H. Mroué, H. P. Pfeiffer, M. A. Scheel, and B. Szilágyi, Phys. Rev. D 89, 084006 (2014).

[42] A. Taracchini et al., Phys. Rev. D 89, 061502 (2014).

[43] S. Babak, A. Taracchini, and A. Buonanno, Phys. Rev. D 95, 024010 (2017).

[44] B. Abbott et al. (LIGO Scientific Collaboration and Virgo Collaboration), Phys. Rev. X 6, 041014 (2016).

[45] F. Hofmann, E. Barausse, and L. Rezzolla, Astrophys. J. Lett. 825, L19 (2016).

[46] X. Jiménez-Forteza, D. Keitel, S. Husa, M. Hannam, S. Khan, and M. Pürrer, Phys. Rev. D 95, 064024 (2017).

[47] J. Healy and C. O. Lousto, Phys. Rev. D 95, 024037 (2017).

[48] N. K. Johnson-McDaniel et al., LIGO Scientic Collaboration and Virgo Collaboration Technical Report No. LIGO-T1600168, 2016 [https://dcc.ligo.org/ LIGO-T1600168/public].

[49] D. Keitel et al., arXiv:1612.09566.

[50] A. Krolak and B. F. Schutz, Gen. Relativ. Gravit. 19, 1163 (1987).

[51] D. E. Holz and S. A. Hughes, Astrophys. J. 629, 15 (2005).

[52] P. A. R. Ade et al. (Planck Collaboration), Astron. Astrophys. 594, A13 (2016).

[53] P. Ajith and S. Bose, Phys. Rev. D 79, 084032 (2009).

[54] J. Veitch, M. Pürrer, and I. Mandel, Phys. Rev. Lett. 115, 141101 (2015).

[55] P. B. Graff, A. Buonanno, and B. S. Sathyaprakash, Phys. Rev. D 92, 022002 (2015).

[56] C.-J. Haster, Z. Wang, C. P. L. Berry, S. Stevenson, J. Veitch, and I. Mandel, Mon. Not. R. Astron. Soc. 457, 4499 (2016).

[57] A. Ghosh, W. Del Pozzo, and P. Ajith, Phys. Rev. D 94, 104070 (2016).

[58] L. S. Finn and D. F. Chernoff, Phys. Rev. D 47, 2198 (1993). 
[59] C. Cutler and É. E. Flanagan, Phys. Rev. D 49, 2658 (1994).

[60] L. Blanchet, T. Damour, B. R. Iyer, C. M. Will, and A. G. Wiseman, Phys. Rev. Lett. 74, 3515 (1995).

[61] S. Vitale, R. Lynch, J. Veitch, V. Raymond, and R. Sturani, Phys. Rev. Lett. 112, 251101 (2014).

[62] T. A. Apostolatos, C. Cutler, G. J. Sussman, and K. S. Thorne, Phys. Rev. D 49, 6274 (1994).

[63] L. Blanchet, Living Rev. Relativ. 17, 2 (2014) [arXiv:1310.1528].

[64] T. Damour, Phys. Rev. D 64, 124013 (2001).

[65] P. Ajith et al., Phys. Rev. Lett. 106, 241101 (2011).

[66] L. Santamaría et al., Phys. Rev. D 82, 064016 (2010).

[67] M. Campanelli, C. O. Lousto, and Y. Zlochower, Phys. Rev. D 74, 041501 (2006).

[68] C. Reisswig, S. Husa, L. Rezzolla, E. N. Dorband, D. Pollney, and J. Seiler, Phys. Rev. D 80, 124026 (2009).

[69] M. Pürrer, M. Hannam, P. Ajith, and S. Husa, Phys. Rev. D 88, 064007 (2013).

[70] M. Pürrer, M. Hannam, and F. Ohme, Phys. Rev. D 93, 084042 (2016).

[71] S. Vitale, R. Lynch, V. Raymond, R. Sturani, J. Veitch, and P. Graff, Phys. Rev. D 95, 064053 (2017).

[72] É. Racine, Phys. Rev. D 78, 044021 (2008).

[73] D. Gerosa, M. Kesden, U. Sperhake, E. Berti, and R. O’Shaughnessy, Phys. Rev. D 92, 064016 (2015).

[74] B. F. Schutz, Nature (London) 323, 310 (1986).

[75] S. Nissanke, D. E. Holz, S. A. Hughes, N. Dalal, and J. L. Sievers, Astrophys. J. 725, 496 (2010).

[76] C. L. Rodriguez, B. Farr, V. Raymond, W. M. Farr, T. B. Littenberg, D. Fazi, and V. Kalogera, Astrophys. J. 784, 119 (2014).

[77] B. Farr et al., Astrophys. J. 825, 116 (2016).

[78] B. P. Abbott et al. (LIGO Scientific Collaboration and Virgo Collaboration), Classical Quantum Gravity 34, 104002 (2017).

[79] B. P. Abbott et al. (LIGO Scientific Collaboration and Virgo Collaboration), Phys. Rev. D 94, 064035 (2016).

[80] V. Varma, P. Ajith, S. Husa, J. C. Bustillo, M. Hannam, and M. Pürrer, Phys. Rev. D 90, 124004 (2014).

[81] J. Calderón Bustillo, S. Husa, A. M. Sintes, and M. Pürrer, Phys. Rev. D 93, 084019 (2016).

[82] N. J. Cornish and T.B. Littenberg, Classical Quantum Gravity 32, 135012 (2015).

[83] E. E. Salpeter, Astrophys. J. 121, 161 (1955).

[84] S. F. Portegies Zwart and S. McMillan, Astrophys. J. Lett. 528, L17 (2000).

[85] C. L. Rodriguez, C.-J. Haster, S. Chatterjee, V. Kalogera, and F. A. Rasio, Astrophys. J. Lett. 824, L8 (2016).

[86] R. M. O'Leary, Y. Meiron, and B. Kocsis, Astrophys. J. Lett. 824, L12 (2016).

[87] M. Mapelli, Mon. Not. R. Astron. Soc. 459, 3432 (2016).

[88] S. Banerjee, Mon. Not. R. Astron. Soc. 467, 524 (2017).

[89] J. R. Hurley, A. C. Sippel, C. A. Tout, and S. J. Aarseth, Pub. Astron. Soc. Aust. 33, e036 (2016).

[90] A. Askar, M. Szkudlarek, D. Gondek-Rosińska, M. Giersz, and T. Bulik, Mon. Not. R. Astron. Soc. Lett. 464, L36 (2017).

[91] D. Park, C. Kim, H. M. Lee, Y.-B. Bae, and C. Belczynski, arXiv:1703.01568 [Mon. Not. R. Astron. Soc. (in press)].
[92] N. C. Stone, B. D. Metzger, and Z. Haiman, Mon. Not. R. Astron. Soc. 464, 946 (2017).

[93] I. Bartos, B. Kocsis, Z. Haiman, and S. Márka, Astrophys. J. 835, 165 (2017).

[94] F. Antonini and F. A. Rasio, Astrophys. J. 831, 187 (2016).

[95] K. Silsbee and S. Tremaine, Astrophys. J. 836, 39 (2017).

[96] F. Antonini, S. Toonen, and A.S. Hamers, arXiv: 1703.06614 [Astrophys. J. (to be published)].

[97] A. Tutukov and L. Yungelson, Nauchnye Informatsii 27, 70 (1973) [http://adsabs.harvard.edu/abs/1973NInfo..27.. .70T].

[98] R. Voss and T. M. Tauris, Mon. Not. R. Astron. Soc. 342 , 1169 (2003).

[99] N. Mennekens and D. Vanbeveren, Astron. Astrophys. 564, A134 (2014).

[100] K. Belczynski, D. E. Holz, T. Bulik, and R. O'Shaughnessy, Nature (London) 534, 512 (2016).

[101] J. J. Eldridge and E. R. Stanway, Mon. Not. R. Astron. Soc. 462, 3302 (2016).

[102] V. M. Lipunov, V. Kornilov, E. Gorbovskoy, N. Tiurina, P. Balanutsa, and A. Kuznetsov, New Astron. 51, 122 (2017).

[103] S. E. Woosley, Astrophys. J. Lett. 824, L10 (2016).

[104] M. U. Kruckow, T. M. Tauris, N. Langer, D. Szécsi, P. Marchant, and P. Podsiadlowski, Astron. Astrophys. 596, A58 (2016).

[105] S. Stevenson, A. Vigna-Gómez, I. Mandel, J. W. Barrett, C. J. Neijssel, D. Perkins, and S.E. de Mink, Nat. Commun. 8, 14906 (2017).

[106] P. Marchant, N. Langer, P. Podsiadlowski, T. M. Tauris, and T. J. Moriya, Astron. Astrophys. 588, A50 (2016).

[107] I. Mandel and S. E. de Mink, Mon. Not. R. Astron. Soc. 458, 2634 (2016).

[108] K. Pavlovskii, N. Ivanova, K. Belczynski, and K. X. Van, Mon. Not. R. Astron. Soc. 465, 2092 (2017).

[109] E. P. J. van den Heuvel, S. F. Portegies Zwart, and S. E. de Mink, arXiv:1701.02355.

[110] T. Hartwig, M. Volonteri, V. Bromm, R. S. Klessen, E. Barausse, M. Magg, and A. Stacy, Mon. Not. R. Astron. Soc. Lett. 460, L74 (2016).

[111] K. Inayoshi, R. Hirai, T. Kinugawa, and K. Hotokezaka, arXiv:1701.04823 [Mon. Not. R. Astron. Soc. (to be published)].

[112] J. S. Vink, New Astron. Rev. 52, 419 (2008).

[113] K. Belczynski, T. Bulik, C. L. Fryer, A. Ruiter, J. S. Vink, and J. R. Hurley, Astrophys. J. 714, 1217 (2010).

[114] M. Spera, M. Mapelli, and A. Bressan, Mon. Not. R. Astron. Soc. 451, 4086 (2015).

[115] C. L. Rodriguez, S. Chatterjee, and F. A. Rasio, Phys. Rev. D 93, 084029 (2016).

[116] V. Petit, Z. Keszthelyi, R. MacInnis, D. H. Cohen, R. H. D. Townsend, G. A. Wade, S. L. Thomas, S. P. Owocki, J. Puls, and J. A. ud-Doula, Mon. Not. R. Astron. Soc. 466, 1052 (2017).

[117] B. J. Carr and S. W. Hawking, Mon. Not. R. Astron. Soc. 168, 399 (1974).

[118] S. Bird, I. Cholis, J. B. Muñoz, Y. Ali-Haïmoud, M. Kamionkowski, E. D. Kovetz, A. Raccanelli, and A. G. Riess, Phys. Rev. Lett. 116, 201301 (2016).

[119] S. Clesse and J. García-Bellido, Phys. Dark Universe 15, 142 (2017). 
[120] B. Carr, F. Kühnel, and M. Sandstad, Phys. Rev. D 94, 083504 (2016).

[121] M. Sasaki, T. Suyama, T. Tanaka, and S. Yokoyama, Phys. Rev. Lett. 117, 061101 (2016).

[122] M. Ricotti, J. P. Ostriker, and K. J. Mack, Astrophys. J. 680, 829 (2008).

[123] S. Clesse and J. García-Bellido, Phys. Rev. D 92, 023524 (2015).

[124] A. Kashlinsky, Astrophys. J. Lett. 823, L25 (2016).

[125] A. M. Green, Phys. Rev. D 94, 063530 (2016).

[126] Y. Ali-Haïmoud and M. Kamionkowski, Phys. Rev. D 95, 043534 (2017).

[127] E. Mediavilla, J. Jiménez-Vicente, J. A. Muñoz, H. VivesArias, and J. Calderón-Infante, Astrophys. J. Lett. 836, L18 (2017).

[128] B. Carr, M. Raidal, T. Tenkanen, V. Vaskonen, and H. Veermäe, arXiv:1705.05567.

[129] C. L. Rodriguez, M. Zevin, C. Pankow, V. Kalogera, and F. A. Rasio, Astrophys. J. Lett. 832, L2 (2016).

[130] V. Kalogera, Astrophys. J. 541, 319 (2000).

[131] J. M. Bardeen and J. A. Petterson, Astrophys. J. Suppl. Ser. 195, L65 (1975).

[132] A. R. King, S. H. Lubow, G. I. Ogilvie, and J. E. Pringle, Mon. Not. R. Astron. Soc. 363, 49 (2005).

[133] J. P. Zahn, Astron. Astrophys. 57, 383 (1977) [http:// adsabs.harvard.edu/abs/1977A\%26A....57..383Z].

[134] P. Hut, Astron. Astrophys. 99, 126 (1981) [http://adsabs .harvard.edu/abs/1981A\%26A....99..126H].

[135] R. O'Shaughnessy, D. Gerosa, and D. Wysocki, arXiv: 1704.03879.

[136] R. G. Martin, C. A. Tout, and J. E. Pringle, Mon. Not. R. Astron. Soc. 401, 1514 (2010).

[137] T.-W. Wong, F. Valsecchi, A. Ansari, T. Fragos, E. Glebbeek, V. Kalogera, and J. McClintock, Astrophys. J. 790, 119 (2014).

[138] H.-T. Janka, Mon. Not. R. Astron. Soc. 434, 1355 (2013).

[139] S. Repetto and G. Nelemans, Mon. Not. R. Astron. Soc. 453, 3341 (2015).

[140] I. Mandel, Mon. Not. R. Astron. Soc. 456, 578 (2016).

[141] S. Repetto, A. P. Igoshev, and G. Nelemans, Mon. Not. R. Astron. Soc. 467, 298 (2017).

[142] M. Dominik, K. Belczynski, C. Fryer, D. E. Holz, E. Berti, T. Bulik, I. Mandel, and R. O'Shaughnessy, Astrophys. J. 779, 72 (2013).

[143] H. Spruit and E. S. Phinney, Nature (London) 393, 139 (1998).

[144] W. M. Farr, K. Kremer, M. Lyutikov, and V. Kalogera, Astrophys. J. 742, 81 (2011).

[145] R. D. Ferdman et al., Astrophys. J. 767, 85 (2013).

[146] R. Kazeroni, J. Guilet, and T. Foglizzo, arXiv:1701.07029.

[147] M. C. Miller and J. M. Miller, Phys. Rep. 548, 1 (2015).

[148] T. Fragos and J. E. McClintock, Astrophys. J. 800, 17 (2015).

[149] J. Fuller, M. Cantiello, D. Lecoanet, and E. Quataert, Astrophys. J. 810, 101 (2015); 815, 137(E) (2015).

[150] M. Zaldarriaga, D. Kushnir, and J. A. Kollmeier, arXiv:1702.00885.
[151] K. Hotokezaka and T. Piran, arXiv:1702.03952.

[152] D. Gerosa, M. Kesden, E. Berti, R. O'Shaughnessy, and U. Sperhake, Phys. Rev. D 87, 104028 (2013).

[153] W. M. Farr, S. Stevenson, M. C. Miller, A. Vecchio, and I. Mandel, Technical Report No. LIGO-P1700067, 2017 [https://dcc.ligo.org/P1700067/public].

[154] D. Gerosa, R. O'Shaughnessy, M. Kesden, E. Berti, and U. Sperhake, Phys. Rev. D 89, 124025 (2014).

[155] S. Vitale, R. Lynch, R. Sturani, and P. Graff, Classical Quantum Gravity 34, 03LT01 (2017).

[156] D. Gerosa and E. Berti, arXiv:1703.06223.

[157] M. Fishbach, D. E. Holz, and B. Farr, Astrophys. J. Lett. 840, L24 (2017).

[158] S. Stevenson, C. P. L. Berry, and I. Mandel, arXiv: 1703.06873

[159] C. Talbot and E. Thrane, arXiv:1704.08370.

[160] S. Mirshekari, N. Yunes, and C. M. Will, Phys. Rev. D 85, 024041 (2012).

[161] N. Yunes, K. Yagi, and F. Pretorius, Phys. Rev. D 94, 084002 (2016).

[162] D. Mattingly, Living Rev. Relativ. 8, 5 (2005) [arXiv:gr-qc/ 0502097].

[163] C. M. Will, Living Rev. Relativ. 17, 4 (2014) [arXiv: 1403.7377].

[164] G. Calcagni, Phys. Rev. Lett. 104, 251301 (2010).

[165] G. Amelino-Camelia, Nature (London) 418, 34 (2002).

[166] P. Hořava, Phys. Rev. D 79, 084008 (2009).

[167] A. S. Sefiedgar, K. Nozari, and H. R. Sepangi, Phys. Lett. B 696, 119 (2011).

[168] M. D. Seifert, Phys. Rev. D 81, 065010 (2010).

[169] B. Altschul, Q. G. Bailey, and V. A. Kostelecky, Phys. Rev. D 81, 065028 (2010).

[170] C. M. Will, Phys. Rev. D 57, 2061 (1998).

[171] L. Blanchet, T. Damour, G. Esposito-Farèse, and B. R. Iyer, Phys. Rev. Lett. 93, 091101 (2004).

[172] L. Blanchet and B.S. Sathyaprakash, Classical Quantum Gravity 11, 2807 (1994).

[173] L. Blanchet, T. Damour, and B. R. Iyer, Phys. Rev. D 51, 5360 (1995); 54, 1860(E) (1996).

[174] K. G. Arun, B. R. Iyer, M. S. S. Qusailah, and B. S. Sathyaprakash, Phys. Rev. D 74, 024006 (2006).

[175] C. K. Mishra, K. G. Arun, B. R. Iyer, and B. S. Sathyaprakash, Phys. Rev. D 82, 064010 (2010).

[176] N. Yunes and F. Pretorius, Phys. Rev. D 80, 122003 (2009).

[177] T. G. F. Li, W. Del Pozzo, S. Vitale, C. Van Den Broeck, M. Agathos, J. Veitch, K. Grover, T. Sidery, R. Sturani, and A. Vecchio, Phys. Rev. D 85, 082003 (2012).

[178] A. Ghosh et al., Phys. Rev. D 94, 021101 (2016).

[179] A. Ghosh, N. K. Johnson-McDaniel, A. Ghosh, C. K. Mishra, P. Ajith, W. Del Pozzo, C. P. L. Berry, A. B. Nielsen, and L. London, arXiv:1704.06784.

[180] LIGO Scientific Collaboration, LIGO Open Science Center release of GW170104, DOI: 10.7935/K53X84K2 (2017). 
B. P. Abbott, ${ }^{1}$ R. Abbott, ${ }^{1}$ T. D. Abbott, ${ }^{2}$ F. Acernese,${ }^{3,4}$ K. Ackley, ${ }^{5}$ C. Adams, ${ }^{6}$ T. Adams, ${ }^{7}$ P. Addesso, ${ }^{8}$ R. X. Adhikari,${ }^{1}$ V. B. Adya, ${ }^{9}$ C. Affeldt, ${ }^{9}$ M. Afrough, ${ }^{10}$ B. Agarwal, ${ }^{11}$ M. Agathos, ${ }^{12}$ K. Agatsuma, ${ }^{13}$ N. Aggarwal, ${ }^{14}$ O. D. Aguiar, ${ }^{15}$ L. Aiello, ${ }^{16,17}$ A. Ain, ${ }^{18}$ P. Ajith, ${ }^{19}$ B. Allen,,${ }^{9,20}$ G. Allen, ${ }^{11}$ A. Allocca, ${ }^{22,23}$ P. A. Altin, ${ }^{24}$ A. Amato, ${ }^{25}$ A. Ananyeva, ${ }^{1}$ S. B. Anderson, ${ }^{1}$ W. G. Anderson, ${ }^{20}$ S. Antier, ${ }^{26}$ S. Appert, ${ }^{1}$ K. Arai, ${ }^{1}$ M. C. Araya, ${ }^{1}$ J. S. Areeda, ${ }^{27}$ N. Arnaud, ${ }^{26,28}$ K. G. Arun, ${ }^{29}$ S. Ascenzi, ${ }^{30,17}$ G. Ashton, ${ }^{9}$ M. Ast ${ }^{31}$ S. M. Aston, ${ }^{6}$ P. Astone,${ }^{32}$ P. Aufmuth,${ }^{21}$ C. Aulbert, ${ }^{9}$ K. AultONeal, ${ }^{33}$ A. Avila-Alvarez, ${ }^{27}$ S. Babak,${ }^{34}$ P. Bacon, ${ }^{35}$ M. K. M. Bader, ${ }^{13}$ S. Bae, ${ }^{36}$ P. T. Baker, ${ }^{37,38}$ F. Baldaccini, ${ }^{39,40}$ G. Ballardin, ${ }^{28}$ S. W. Ballmer, ${ }^{41}$ S. Banagiri, ${ }^{42}$ J. C. Barayoga, ${ }^{1}$ S. E. Barclay, ${ }^{43}$ B. C. Barish, ${ }^{1}$ D. Barker ${ }^{44}$ F. Barone,${ }^{3,4}$ B. Barr, ${ }^{43}$ L. Barsotti ${ }^{14}$ M. Barsuglia, ${ }^{35}$ D. Barta,${ }^{45}$ J. Bartlett, ${ }^{44}$ I. Bartos, ${ }^{46}$ R. Bassiri,${ }^{47}$ A. Basti, ${ }^{22,23}$ J. C. Batch, ${ }^{44}$ C. Baune, ${ }^{9}$ M. Bawaj, ${ }^{48,40}$ M. Bazzan, ${ }^{49,50}$ B. Bécsy, ${ }^{51}$ C. Beer, ${ }^{9}$ M. Bejger, ${ }^{52}$ I. Belahcene,${ }^{26}$ A. S. Bell, ${ }^{43}$ B. K. Berger, ${ }^{1}$ G. Bergmann, ${ }^{9}$ C. P. L. Berry, ${ }^{53}$ D. Bersanetti, ${ }^{54,55}$ A. Bertolini, ${ }^{13}$ J. Betzwieser, ${ }^{6}$ S. Bhagwat, ${ }^{41}$ R. Bhandare, ${ }^{56}$ I. A. Bilenko, ${ }^{57}$ G. Billingsley, ${ }^{1}$ C. R. Billman, ${ }^{5}$ J. Birch,${ }^{6}$ R. Birney,${ }^{58}$ O. Birnholtz, ${ }^{9}$ S. Biscans,${ }^{14}$ A. Bisht,${ }^{21}$ M. Bitossi,${ }^{28,23}$ C. Biwer,${ }^{41}$ M. A. Bizouard, ${ }^{26}$ J. K. Blackburn, ${ }^{1}$ J. Blackman, ${ }^{59}$ C. D. Blair, ${ }^{60}$ D. G. Blair, ${ }^{60}$ R. M. Blair ${ }^{44}$ S. Bloemen, ${ }^{61}$ O. Bock, ${ }^{9}$ N. Bode, ${ }^{9}$ M. Boer,${ }^{62}$ G. Bogaert, ${ }^{62}$ A. Bohe, ${ }^{34}$ F. Bondu, ${ }^{63}$ R. Bonnand, ${ }^{7}$ B. A. Boom, ${ }^{13}$ R. Bork, ${ }^{1}$ V. Boschi, ${ }^{22,23}$ S. Bose ${ }^{64,18}$ Y. Bouffanais, ${ }^{35}$ A. Bozzi, ${ }^{28}$ C. Bradaschia, ${ }^{23}$ P. R. Brady,${ }^{20}$ V. B. Braginsky,${ }^{57, \dagger}$ M. Branchesi, ${ }^{65,66}$ J. E. Brau, ${ }^{67}$ T. Briant, ${ }^{68}$ A. Brillet, ${ }^{62}$ M. Brinkmann, ${ }^{9}$ V. Brisson, ${ }^{26}$ P. Brockill, ${ }^{20}$ J. E. Broida, ${ }^{69}$ A. F. Brooks, ${ }^{1}$ D. A. Brown, ${ }^{41}$ D. D. Brown ${ }^{53}$ N. M. Brown, ${ }^{14}$ S. Brunett, ${ }^{1}$ C. C. Buchanan, ${ }^{2}$ A. Buikema, ${ }^{14}$ T. Bulik, ${ }^{70}$ H. J. Bulten, ${ }^{71,13}$ A. Buonanno, ${ }^{34,72}$ D. Buskulic, ${ }^{7}$ C. Buy,${ }^{35}$ R. L. Byer, ${ }^{47}$ M. Cabero, ${ }^{9}$ L. Cadonati, ${ }^{73}$ G. Cagnoli, ${ }^{25,74}$ C. Cahillane, ${ }^{1}$ J. Calderón Bustillo, ${ }^{73}$ T. A. Callister, ${ }^{1}$ E. Calloni, ${ }^{75,4}$ J. B. Camp,${ }^{76}$ M. Canepa,${ }^{54,55}$ P. Canizares, ${ }^{61}$ K. C. Cannon, ${ }^{77}$ H. Cao, ${ }^{78}$ J. Cao, ${ }^{79}$ C. D. Capano, ${ }^{9}$ E. Capocasa, ${ }^{35}$ F. Carbognani, ${ }^{28}$ S. Caride ${ }^{80}$ M. F. Carney, ${ }^{81}$ J. Casanueva Diaz,${ }^{26}$ C. Casentini,${ }^{30,17}$ S. Caudill, ${ }^{20}$ M. Cavaglià, ${ }^{10}$ F. Cavalier, ${ }^{26}$ R. Cavalieri, ${ }^{28}$ G. Cella, ${ }^{23}$ C. B. Cepeda, ${ }^{1}$ L. Cerboni Baiardi, ${ }^{65,66}$ G. Cerretani, ${ }^{22,23}$ E. Cesarini, ${ }^{30,17}$ S. J. Chamberlin, ${ }^{82}$ M. Chan, ${ }^{43}$ S. Chao, ${ }^{83}$ P. Charlton, ${ }^{84}$ E. Chassande-Mottin, ${ }^{35}$ D. Chatterjee, ${ }^{20}$ K. Chatziioannou, ${ }^{85}$ B. D. Cheeseboro, ${ }^{37,38}$ H. Y. Chen,${ }^{86}$ Y. Chen, ${ }^{59}$ H.-P. Cheng, ${ }^{5}$ A. Chincarini, ${ }^{55}$ A. Chiummo, ${ }^{28}$ T. Chmiel, ${ }^{81}$ H. S. Cho ${ }^{87}$ M. Cho, ${ }^{72}$ J. H. Chow,${ }^{24}$ N. Christensen,${ }^{69,62}$ Q. Chu, ${ }^{60}$ A. J. K. Chua,${ }^{12}$ S. Chua,${ }^{68}$ A. K. W. Chung ${ }^{88}$ S. Chung, ${ }^{60}$ G. Ciani, ${ }^{5}$ R. Ciolfi, ${ }^{89,90}$ C. E. Cirelli, ${ }^{47}$ A. Cirone,${ }^{54,55}$ F. Clara, ${ }^{44}$ J. A. Clark, ${ }^{73}$ F. Cleva, ${ }^{62}$ C. Cocchieri, ${ }^{10}$ E. Coccia, ${ }^{16,17}$ P.-F. Cohadon, ${ }^{68}$ A. Colla, ${ }^{91,32}$ C. G. Collette, ${ }^{92}$ L. R. Cominsky, ${ }^{93}$ M. Constancio Jr., ${ }^{15}$ L. Conti, ${ }^{50}$ S. J. Cooper, ${ }^{53}$ P. Corban, ${ }^{6}$ T. R. Corbitt, ${ }^{2}$ K. R. Corley ${ }^{46}$ N. Cornish, ${ }^{94}$ A. Corsi, ${ }^{80}$ S. Cortese,${ }^{28}$ C. A. Costa, ${ }^{15}$ M. W. Coughlin, ${ }^{69}$ S. B. Coughlin, ${ }^{95,96}$ J.-P. Coulon, ${ }^{62}$ S. T. Countryman, ${ }^{46}$ P. Couvares, ${ }^{1}$ P. B. Covas, ${ }^{97}$ E. E. Cowan, ${ }^{73}$

D. M. Coward, ${ }^{60}$ M. J. Cowart, ${ }^{6}$ D. C. Coyne, ${ }^{1}$ R. Coyne, ${ }^{80}$ J. D. E. Creighton, ${ }^{20}$ T. D. Creighton, ${ }^{98}$ J. Cripe, ${ }^{2}$

S. G. Crowder, ${ }^{99}$ T. J. Cullen, ${ }^{27}$ A. Cumming, ${ }^{43}$ L. Cunningham, ${ }^{43}$ E. Cuoco,${ }^{28}$ T. Dal Canton, ${ }^{76}$ S. L. Danilishin, ${ }^{21,9}$ S. D’Antonio, ${ }^{17}$ K. Danzmann, ${ }^{21,9}$ A. Dasgupta, ${ }^{100}$ C. F. Da Silva Costa, ${ }^{5}$ V. Dattilo, ${ }^{28}$ I. Dave, ${ }^{56}$ M. Davier, ${ }^{26}$ D. Davis, ${ }^{41}$ E. J. Daw, ${ }^{101}$ B. Day, ${ }^{73}$ S. De,${ }^{41}$ D. DeBra, ${ }^{47}$ E. Deelman, ${ }^{102}$ J. Degallaix,${ }^{25}$ M. De Laurentis ${ }^{75,4}$ S. Deléglise ${ }^{68}$ W. Del Pozzo, ${ }^{53,22,23}$ T. Denker, ${ }^{9}$ T. Dent, ${ }^{9}$ V. Dergachev,${ }^{34}$ R. De Rosa,${ }^{75,4}$ R. T. DeRosa, ${ }^{6}$ R. DeSalvo, ${ }^{103}$ J. Devenson, ${ }^{58}$ R. C. Devine,${ }^{37,38}$ S. Dhurandhar ${ }^{18}$ M. C. Díaz, ${ }^{98}$ L. Di Fiore, ${ }^{4}$ M. Di Giovanni, ${ }^{104,90}$ T. Di Girolamo, ${ }^{75,446}$ A. Di Lieto, ${ }^{22,23}$ S. Di Pace, ${ }^{91,32}$ I. Di Palma, ${ }^{91,32}$ F. Di Renzo, ${ }^{22,23}$ Z. Doctor, ${ }^{86}$ V. Dolique, ${ }^{25}$ F. Donovan, ${ }^{14}$ K. L. Dooley, ${ }^{10}$ S. Doravari, ${ }^{9}$ I. Dorrington, ${ }^{96}$ R. Douglas, ${ }^{43}$ M. Dovale Álvarez,${ }^{53}$ T. P. Downes, ${ }^{20}$ M. Drago, ${ }^{9}$ R. W. P. Drever, ${ }^{1,}$ J. C. Driggers, ${ }^{44}$ Z. Du, ${ }^{79}$ M. Ducrot, ${ }^{7}$ J. Duncan, ${ }^{95}$ S. E. Dwyer, ${ }^{44}$ T. B. Edo, ${ }^{101}$ M. C. Edwards, ${ }^{69}$ A. Effler, ${ }^{6}$ H.-B. Eggenstein, ${ }^{9}$ P. Ehrens, ${ }^{1}$ J. Eichholz, ${ }^{1}$ S. S. Eikenberry, ${ }^{5}$ R. A. Eisenstein, ${ }^{14}$ R. C. Essick, ${ }^{14}$ Z. B. Etienne, ${ }^{37,38}$ T. Etzel, ${ }^{1}$ M. Evans, ${ }^{14}$ T. M. Evans, ${ }^{6}$ M. Factourovich, ${ }^{46}$ V. Fafone, ${ }^{30,17,16}$ H. Fair, ${ }^{41}$ S. Fairhurst, ${ }^{96}$ X. Fan, ${ }^{79}$ S. Farinon, ${ }^{55}$ B. Farr, ${ }^{86}$ W. M. Farr, ${ }^{53}$ E. J. Fauchon-Jones, ${ }^{96}$ M. Favata, ${ }^{105}$ M. Fays, ${ }^{96}$ H. Fehrmann, ${ }^{9}$ J. Feicht, ${ }^{1}$ M. M. Fejer, ${ }^{47}$ A. Fernandez-Galiana, ${ }^{14}$ I. Ferrante, ${ }^{22,23}$ E. C. Ferreira, ${ }^{15}$ F. Ferrini, ${ }^{28}$ F. Fidecaro, ${ }^{22,23}$ I. Fiori, ${ }^{28}$ D. Fiorucci, ${ }^{35}$ R. P. Fisher, ${ }^{41}$ R. Flaminio, ${ }^{25,106}$ M. Fletcher, ${ }^{43}$ H. Fong, ${ }^{85}$ P. W. F. Forsyth, ${ }^{24}$ S. S. Forsyth, ${ }^{73}$ J.-D. Fournier, ${ }^{62}$ S. Frasca, ${ }^{91,32}$ F. Frasconi, ${ }^{23}$ Z. Frei ${ }^{51}$ A. Freise,${ }^{53}$ R. Frey,${ }^{67}$ V. Frey, ${ }^{26}$ E. M. Fries,${ }^{1}$ P. Fritschel, ${ }^{14}$ V. V. Frolov,${ }^{6}$ P. Fulda, ${ }^{5,76}$ M. Fyffe,${ }^{6}$ H. Gabbard,${ }^{9}$ M. Gabel, ${ }^{107}$ B. U. Gadre, ${ }^{18}$ S. M. Gaebel, ${ }^{53}$ J. R. Gair, ${ }^{108}$ L. Gammaitoni, ${ }^{39}$ M. R. Ganija, ${ }^{78}$ S. G. Gaonkar, ${ }^{18}$ F. Garufi, ${ }^{75}, 4$ S. Gaudio, ${ }^{33}$ G. Gaur, ${ }^{109}$ V. Gayathri, ${ }^{110}$ N. Gehrels, ${ }^{76,8}$ G. Gemme, ${ }^{55}$ E. Genin, ${ }^{28}$ A. Gennai, ${ }^{23}$ D. George,${ }^{11}$ J. George,${ }^{56}$ L. Gergely, ${ }^{111}$ V. Germain, ${ }^{7}$ S. Ghonge, ${ }^{73}$ Abhirup Ghosh, ${ }^{19}$ Archisman Ghosh, ${ }^{19,13}$ S. Ghosh,${ }^{61,13}$ J. A. Giaime, ${ }^{2,6}$ K. D. Giardina, ${ }^{6}$ A. Giazotto, ${ }^{23}$ K. Gill, ${ }^{33}$ L. Glover, ${ }^{103}$ E. Goetz, ${ }^{9}$ R. Goetz, ${ }^{5}$ S. Gomes,${ }^{96}$ G. González, ${ }^{2}$ J. M. Gonzalez Castro, ${ }^{22,23}$ A. Gopakumar, ${ }^{112}$ M. L. Gorodetsky, ${ }^{57}$ S. E. Gossan, ${ }^{1}$ M. Gosselin, ${ }^{28}$ R. Gouaty, ${ }^{7}$ A. Grado,,${ }^{113,4}$ C. Graef, ${ }^{43}$ M. Granata ${ }^{25}$ A. Grant, ${ }^{43}$ S. Gras, ${ }^{14}$ C. Gray, ${ }^{44}$ G. Greco, ${ }^{65,66}$ A. C. Green, ${ }^{53}$ P. Groot, ${ }^{61}$ H. Grote, ${ }^{9}$ S. Grunewald, ${ }^{34}$ P. Gruning, ${ }^{26}$ G. M. Guidi ${ }^{65,66}$ X. Guo, ${ }^{79}$ A. Gupta, ${ }^{82}$ M. K. Gupta, ${ }^{100}$ K. E. Gushwa, ${ }^{1}$ E. K. Gustafson, ${ }^{1}$ R. Gustafson, ${ }^{114}$ B. R. Hall, ${ }^{64}$ 
E. D. Hall, ${ }^{1}$ G. Hammond, ${ }^{43}$ M. Haney, ${ }^{112}$ M. M. Hanke, ${ }^{9}$ J. Hanks, ${ }^{44}$ C. Hanna,${ }^{82}$ M. D. Hannam, ${ }^{96}$ O. A. Hannuksela ${ }^{88}$ J. Hanson, ${ }^{6}$ T. Hardwick, ${ }^{2}$ J. Harms, ${ }^{65,66}$ G. M. Harry, ${ }^{115}$ I. W. Harry ${ }^{34}$ M. J. Hart, ${ }^{43}$ C.-J. Haster, ${ }^{85}$ K. Haughian, ${ }^{43}$

J. Healy, ${ }^{116}$ A. Heidmann, ${ }^{68}$ M. C. Heintze,${ }^{6}$ H. Heitmann, ${ }^{62}$ P. Hello, ${ }^{26}$ G. Hemming, ${ }^{28}$ M. Hendry, ${ }^{43}$ I. S. Heng, ${ }^{43}$ J. Hennig, ${ }^{43}$ J. Henry, ${ }^{116}$ A. W. Heptonstall, ${ }^{1}$ M. Heurs, ${ }^{9,21}$ S. Hild, ${ }^{43}$ D. Hoak,${ }^{28}$ D. Hofman, ${ }^{25}$ K. Holt, ${ }^{6}$ D. E. Holz, ${ }^{86}$ P. Hopkins,${ }^{96}$ C. Horst,${ }^{20}$ J. Hough ${ }^{43}$ E. A. Houston, ${ }^{43}$ E. J. Howell,${ }^{60}$ Y. M. Hu, ${ }^{9}$ E. A. Huerta,${ }^{11}$ D. Huet,${ }^{26}$ B. Hughey,${ }^{33}$ S. Husa, ${ }^{97}$ S. H. Huttner, ${ }^{43}$ T. Huynh-Dinh, ${ }^{6}$ N. Indik,,${ }^{9}$ D. R. Ingram,${ }^{44}$ R. Inta ${ }^{80}$ G. Intini, ${ }^{91,32}$ H. N. Isa, ${ }^{43}$ J.-M. Isac, ${ }^{68}$ M. Isi ${ }^{1}$ B. R. Iyer, ${ }^{19}$ K. Izumi, ${ }^{44}$ T. Jacqmin,${ }^{68}$ K. Jani ${ }^{73}$ P. Jaranowski, ${ }^{117}$ S. Jawahar, ${ }^{118}$ F. Jiménez-Forteza, ${ }^{97}$ W. W. Johnson, ${ }^{2}$ N. K. Johnson-McDaniel, ${ }^{19}$ D. I. Jones, ${ }^{119}$ R. Jones, ${ }^{43}$ R. J. G. Jonker, ${ }^{13}$ L. Ju, ${ }^{60}$ J. Junker, ${ }^{9}$ C. V. Kalaghatgi, ${ }^{96}$ V. Kalogera, ${ }^{95}$ S. Kandhasamy, ${ }^{6}$ G. Kang, ${ }^{36}$ J. B. Kanner, ${ }^{1}$ S. Karki,${ }^{67}$ K. S. Karvinen, ${ }^{9}$ M. Kasprzack, ${ }^{2}$ M. Katolik, ${ }^{11}$ E. Katsavounidis, ${ }^{14}$ W. Katzman, ${ }^{6}$ S. Kaufer, ${ }^{21}$ K. Kawabe, ${ }^{44}$ F. Kéfélian, ${ }^{62}$ D. Keitel, ${ }^{43}$ A. J. Kemball, ${ }^{11}$ R. Kennedy, ${ }^{101}$ C. Kent, ${ }^{96}$ J. S. Key, ${ }^{120}$ F. Y. Khalili, ${ }^{57}$ I. Khan, ${ }^{16,17}$ S. Khan, ${ }^{9}$ Z. Khan, ${ }^{100}$ E. A. Khazanov, ${ }^{121}$ N. Kijbunchoo, ${ }^{44}$ Chunglee Kim, ${ }^{122}$ J. C. Kim, ${ }^{123}$ W. Kim, ${ }^{78}$ W. S. Kim, ${ }^{124}$ Y.-M. Kim, ${ }^{87,122}$ S. J. Kimbrell, ${ }^{73}$ E. J. King, ${ }^{78}$ P. J. King, ${ }^{44}$ R. Kirchhoff, ${ }^{9}$ J. S. Kissel,${ }^{44}$ L. Kleybolte, ${ }^{31}$ S. Klimenko, ${ }^{5}$ P. Koch, ${ }^{9}$ S. M. Koehlenbeck, ${ }^{9}$ S. Koley, ${ }^{13}$ V. Kondrashov, ${ }^{1}$ A. Kontos, ${ }^{14}$ M. Korobko, ${ }^{31}$ W. Z. Korth, ${ }^{1}$ I. Kowalska, ${ }^{70}$ D. B. Kozak, ${ }^{1}$ C. Krämer, ${ }^{9}$ V. Kringel, ${ }^{9}$ B. Krishnan, ${ }^{9}$ A. Królak, ${ }^{125,126}$ G. Kuehn, ${ }^{9}$ P. Kumar, ${ }^{85}$ R. Kumar, ${ }^{100}$ S. Kumar, ${ }^{19}$ L. Kuo, ${ }^{83}$ A. Kutynia, ${ }^{125}$ S. Kwang, ${ }^{20}$ B. D. Lackey, ${ }^{34}$ K. H. Lai ${ }^{88}$ M. Landry, ${ }^{44}$ R. N. Lang, ${ }^{20}$ J. Lange, ${ }^{116}$ B. Lantz, ${ }^{47}$ R. K. Lanza, ${ }^{14}$ A. Lartaux-Vollard, ${ }^{26}$ P. D. Lasky, ${ }^{127}$ M. Laxen, ${ }^{6}$ A. Lazzarini, ${ }^{1}$ C. Lazzaro ${ }^{50}$ P. Leaci, ${ }^{91,32}$ S. Leavey,${ }^{43}$ C. H. Lee, ${ }^{87}$ H. K. Lee,${ }^{128}$ H. M. Lee,${ }^{122}$ H. W. Lee, ${ }^{123}$ K. Lee, ${ }^{43}$ J. Lehmann, ${ }^{9}$ A. Lenon, ${ }^{37,38}$ M. Leonardi, ${ }^{104,90}$ N. Leroy, ${ }^{26}$ N. Letendre, ${ }^{7}$ Y. Levin, ${ }^{127}$ T. G. F. Li,${ }^{88}$ A. Libson, ${ }^{14}$ T. B. Littenberg, ${ }^{129}$ J. Liu, ${ }^{60}$ R. K. L. Lo, ${ }^{88}$ N. A. Lockerbie, ${ }^{118}$ L. T. London, ${ }^{96}$ J. E. Lord, ${ }^{41}$ M. Lorenzini,,${ }^{16,17}$ V. Loriette, ${ }^{130}$ M. Lormand, ${ }^{6}$ G. Losurdo, ${ }^{23}$ J. D. Lough,,${ }^{9,21}$ G. Lovelace, ${ }^{27}$ H. Lück, ${ }^{21,9}$ D. Lumaca, ${ }^{30,17}$ A. P. Lundgren, ${ }^{9}$ R. Lynch, ${ }^{14}$ Y. Ma, ${ }^{59}$ S. Macfoy, ${ }^{58}$ B. Machenschalk, ${ }^{9}$ M. MacInnis, ${ }^{14}$ D. M. Macleod, ${ }^{2}$ I. Magaña Hernandez, ${ }^{88}$ F. Magaña-Sandoval, ${ }^{41}$ L. Magaña Zertuche,${ }^{41}$ R. M. Magee, ${ }^{82}$ E. Majorana, ${ }^{32}$ I. Maksimovic, ${ }^{130}$ N. Man ${ }^{62}$ V. Mandic, ${ }^{42}$ V. Mangano, ${ }^{43}$ G. L. Mansell, ${ }^{24}$ M. Manske,${ }^{20}$ M. Mantovani, ${ }^{28}$ F. Marchesoni, ${ }^{48,40}$ F. Marion, ${ }^{7}$ S. Márka, ${ }^{46}$ Z. Márka, ${ }^{46}$ C. Markakis, ${ }^{11}$ A. S. Markosyan, ${ }^{47}$ E. Maros, ${ }^{1}$ F. Martelli, ${ }^{65,66}$ L. Martellini, ${ }^{62}$ I. W. Martin, ${ }^{43}$ D. V. Martynov, ${ }^{14}$ J. N. Marx, ${ }^{1}$ K. Mason, ${ }^{14}$ A. Masserot, ${ }^{7}$ T. J. Massinger, ${ }^{1}$ M. Masso-Reid, ${ }^{43}$ S. Mastrogiovanni, ${ }^{91,32}$ A. Matas, ${ }^{42}$ F. Matichard, ${ }^{14}$ L. Matone, ${ }^{46}$ N. Mavalvala, ${ }^{14}$ R. Mayani, ${ }^{102}$ N. Mazumder,${ }^{64}$ R. McCarthy,${ }^{44}$ D. E. McClelland, ${ }^{24}$ S. McCormick, ${ }^{6}$ L. McCuller, ${ }^{14}$ S. C. McGuire, ${ }^{131}$ G. McIntyre, ${ }^{1}$ J. McIver, ${ }^{1}$ D. J. McManus, ${ }^{24}$ T. McRae, ${ }^{24}$ S. T. McWilliams, ${ }^{37,38}$ D. Meacher ${ }^{82}$ G. D. Meadors ${ }^{34,9}$ J. Meidam, ${ }^{13}$ E. Mejuto-Villa, ${ }^{8}$ A. Melatos, ${ }^{132}$ G. Mendell, ${ }^{44}$ R. A. Mercer, ${ }^{20}$ E. L. Merilh, ${ }^{44}$ M. Merzougui, ${ }^{62}$ S. Meshkov, ${ }^{1}$ C. Messenger, ${ }^{43}$ C. Messick, ${ }^{82}$ R. Metzdorff, ${ }^{68}$ P. M. Meyers, ${ }^{42}$ F. Mezzani, ${ }^{32,91}$ H. Miao, ${ }^{53}$ C. Michel,${ }^{25}$ H. Middleton, ${ }^{53}$ E. E. Mikhailov, ${ }^{133}$ L. Milano, ${ }^{75,4}$ A. L. Miller, ${ }^{5}$ A. Miller,${ }^{91,32}$ B. B. Miller ${ }^{95}$ J. Miller, ${ }^{14}$ M. Millhouse, ${ }^{94}$ O. Minazzoli, ${ }^{62}$ Y. Minenkov,${ }^{17}$ J. Ming, ${ }^{34}$ C. Mishra, ${ }^{134}$ S. Mitra, ${ }^{18}$ V. P. Mitrofanov, ${ }^{57}$ G. Mitselmakher, ${ }^{5}$ R. Mittleman, ${ }^{14}$ A. Moggi,${ }^{23}$ M. Mohan, ${ }^{28}$ S. R. P. Mohapatra, ${ }^{14}$ M. Montani, ${ }^{65,66}$ B. C. Moore ${ }^{105}$ C. J. Moore,${ }^{12}$ D. Moraru, ${ }^{44}$ G. Moreno, ${ }^{44}$ S. R. Morriss, ${ }^{98}$ B. Mours, ${ }^{7}$ C. M. Mow-Lowry, ${ }^{53}$ G. Mueller, ${ }^{5}$ A. W. Muir, ${ }^{96}$ Arunava Mukherjee, ${ }^{9}$ D. Mukherjee, ${ }^{20}$ S. Mukherjee, ${ }^{98}$ N. Mukund, ${ }^{18}$ A. Mullavey, ${ }^{6}$ J. Munch, ${ }^{78}$ E. A. M. Muniz, ${ }^{41}$ P. G. Murray, ${ }^{43}$ K. Napier, ${ }^{73}$ I. Nardecchia,${ }^{30,17}$ L. Naticchioni, ${ }^{91,32}$ R. K. Nayak, ${ }^{135}$ G. Nelemans, ${ }^{61,13}$ T. J. N. Nelson, ${ }^{6}$ M. Neri, ${ }^{54,55}$ M. Nery, ${ }^{9}$ A. Neunzert, ${ }^{114}$ J. M. Newport, ${ }^{115}$ G. Newton, ${ }^{43, \|}$ K. K. Y. Ng, ${ }^{88}$ T. T. Nguyen, ${ }^{24}$ D. Nichols, ${ }^{61}$ A. B. Nielsen, ${ }^{9}$ S. Nissanke, ${ }^{61,13}$ A. Nitz, ${ }^{9}$ A. Noack, ${ }^{9}$ F. Nocera, ${ }^{28}$ D. Nolting, ${ }^{6}$ M. E. N. Normandin, ${ }^{98}$ L. K. Nuttall, ${ }^{41}$ J. Oberling, ${ }^{44}$ E. Ochsner, ${ }^{20}$ E. Oelker, ${ }^{14}$ G. H. Ogin,,${ }^{107}$ J. J. Oh, ${ }^{124}$ S. H. Oh, ${ }^{124}$ F. Ohme, ${ }^{9}$ M. Oliver, ${ }^{97}$ P. Oppermann, ${ }^{9}$ Richard J. Oram, ${ }^{6}$ B. O'Reilly, ${ }^{6}$ R. Ormiston, ${ }^{42}$ L. F. Ortega, ${ }^{5}$ R. O'Shaughnessy, ${ }^{116}$ D. J. Ottaway, ${ }^{78}$ H. Overmier, ${ }^{6}$ B. J. Owen, ${ }^{80}$ A. E. Pace,${ }^{82}$ J. Page, ${ }^{129}$ M. A. Page ${ }^{60}$ A. Pai ${ }^{110}$ S. A. Pai, ${ }^{56}$ J. R. Palamos,${ }^{67}$ O. Palashov, ${ }^{121}$ C. Palomba, ${ }^{32}$ A. Pal-Singh, ${ }^{31}$ H. Pan, ${ }^{83}$ B. Pang, ${ }^{59}$ P. T. H. Pang, ${ }^{88}$ C. Pankow, ${ }^{95}$ F. Pannarale, ${ }^{96}$ B. C. Pant,${ }^{56}$ F. Paoletti, ${ }^{23}$ A. Paoli, ${ }^{28}$ M. A. Papa,${ }^{34,20,9}$ H. R. Paris, ${ }^{47}$ W. Parker, ${ }^{6}$ D. Pascucci ${ }^{43}$ A. Pasqualetti, ${ }^{28}$ R. Passaquieti, ${ }^{22,23}$ D. Passuello, ${ }^{23}$ B. Patricelli, ${ }_{136,23}$ B. L. Pearlstone,${ }^{43}$ M. Pedraza, ${ }^{1}$ R. Pedurand, ${ }^{25,137}$ L. Pekowsky, ${ }^{41}$ A. Pele, ${ }^{6}$ S. Penn,${ }^{138}$ C. J. Perez, ${ }^{44}$ A. Perreca, ${ }^{1,104,90}$ L. M. Perri, ${ }^{95}$ H. P. Pfeiffer,${ }^{85}$ M. Phelps,${ }^{43}$ O. J. Piccinni, ${ }^{91,32}$ M. Pichot,${ }^{62}$ F. Piergiovanni,${ }^{65,66}$ V. Pierro, ${ }^{8}$ G. Pillant, ${ }^{28}$ L. Pinard, ${ }^{25}$ I. M. Pinto, ${ }^{8}$ M. Pitkin, ${ }^{43}$ R. Poggiani, ${ }^{22,23}$ P. Popolizio, ${ }^{28}$ E. K. Porter,${ }^{35}$ A. Post, ${ }^{9}$ J. Powell, ${ }^{43}$ J. Prasad, ${ }^{18}$ J. W. W. Pratt, ${ }^{33}$ V. Predoi, ${ }^{96}$ T. Prestegard, ${ }^{20}$ M. Prijatelj, ${ }^{9}$ M. Principe, ${ }^{8}$ S. Privitera, ${ }^{34}$ G. A. Prodi, ${ }^{104,90}$ L. G. Prokhorov ${ }^{57}$ O. Puncken, ${ }^{9}$ M. Punturo, ${ }^{40}$ P. Puppo, ${ }^{32}$ M. Pürrer, ${ }^{34}$ H. Qi,${ }^{20}$ J. Qin, ${ }^{60}$ S. Qiu, ${ }^{127}$ V. Quetschke, ${ }^{98}$ E. A. Quintero, ${ }^{1}$ R. Quitzow-James, ${ }^{67}$ F. J. Raab, ${ }^{44}$ D. S. Rabeling,${ }^{24}$ H. Radkins, ${ }^{44}$ P. Raffai,${ }^{51}$ S. Raja ${ }^{56}$ C. Rajan, ${ }^{56}$ M. Rakhmanov, ${ }^{98}$ K. E. Ramirez, ${ }^{98}$ P. Rapagnani, ${ }^{91,32}$ V. Raymond, ${ }^{34}$ M. Razzano, ${ }^{22,23}$ J. Read, ${ }^{27}$ T. Regimbau, ${ }^{62}$ L. Rei, ${ }^{55}$ 
S. Reid ${ }^{58}$ D. H. Reitze, ${ }^{1,5}$ H. Rew, ${ }^{133}$ S. D. Reyes,${ }^{41}$ F. Ricci, ${ }^{91,32}$ P. M. Ricker, ${ }^{11}$ S. Rieger, ${ }^{9}$ K. Riles, ${ }^{114}$ M. Rizzo, ${ }^{116}$ N. A. Robertson, ${ }^{1,43}$ R. Robie, ${ }^{43}$ F. Robinet, ${ }^{26}$ A. Rocchi, ${ }^{17}$ L. Rolland, ${ }^{7}$ J. G. Rollins, ${ }^{1}$ V. J. Roma, ${ }^{67}$ J. D. Romano, ${ }^{98}$

R. Romano, ${ }^{3,4}$ C. L. Romel, ${ }^{44}$ J. H. Romie, ${ }^{6}$ D. Rosińska, ${ }^{139,52}$ M. P. Ross, ${ }^{140}$ S. Rowan, ${ }^{43}$ A. Rüdiger, ${ }^{9}$ P. Ruggi, ${ }^{28}$

K. Ryan, ${ }^{44}$ M. Rynge, ${ }^{102}$ S. Sachdev, ${ }^{1}$ T. Sadecki, ${ }^{44}$ L. Sadeghian, ${ }^{20}$ M. Sakellariadou, ${ }^{141}$ L. Salconi, ${ }^{28}$ M. Saleem, ${ }^{110}$

F. Salemi, ${ }^{9}$ A. Samajdar, ${ }^{135}$ L. Sammut, ${ }^{127}$ L. M. Sampson, ${ }^{95}$ E. J. Sanchez, ${ }^{1}$ V. Sandberg, ${ }^{44}$ B. Sandeen, ${ }^{95}$ J. R. Sanders,${ }^{41}$

B. Sassolas, ${ }^{25}$ B. S. Sathyaprakash, ${ }^{82,96}$ P. R. Saulson, ${ }^{41}$ O. Sauter, ${ }^{114}$ R. L. Savage, ${ }^{44}$ A. Sawadsky, ${ }^{21}$ P. Schale, ${ }^{67}$

J. Scheuer, ${ }^{95}$ E. Schmidt, ${ }^{33}$ J. Schmidt, ${ }^{9}$ P. Schmidt,${ }^{1,61}$ R. Schnabel, ${ }^{31}$ R. M. S. Schofield, ${ }^{67}$ A. Schönbeck, ${ }^{31}$ E. Schreiber, ${ }^{9}$

D. Schuette, ${ }^{9,21}$ B. W. Schulte, ${ }^{9}$ B. F. Schutz, ${ }^{96,9}$ S. G. Schwalbe, ${ }^{33}$ J. Scott,${ }^{43}$ S. M. Scott, ${ }^{24}$ E. Seidel, ${ }^{11}$ D. Sellers,${ }^{6}$

A. S. Sengupta, ${ }^{142}$ D. Sentenac, ${ }^{28}$ V. Sequino, ${ }^{30,17}$ A. Sergeev, ${ }^{121}$ D. A. Shaddock, ${ }^{24}$ T. J. Shaffer, ${ }^{44}$ A. A. Shah, ${ }^{129}$

M. S. Shahriar, ${ }^{95}$ L. Shao, ${ }^{34}$ B. Shapiro, ${ }^{47}$ P. Shawhan, ${ }^{72}$ A. Sheperd, ${ }^{20}$ D. H. Shoemaker, ${ }^{14}$ D. M. Shoemaker, ${ }^{73}$ K. Siellez, ${ }^{73}$

X. Siemens, ${ }^{20}$ M. Sieniawska, ${ }^{52}$ D. Sigg, ${ }^{44}$ A. D. Silva, ${ }^{15}$ A. Singer, ${ }^{1}$ L. P. Singer,${ }^{76}$ A. Singh,${ }^{34,9,21}$ R. Singh, ${ }^{2}$

A. Singhal, ${ }^{16,32}$ A. M. Sintes ${ }^{97}$ B. J. J. Slagmolen, ${ }^{24}$ B. Smith, ${ }^{6}$ J. R. Smith, ${ }^{27}$ R. J. E. Smith, ${ }^{1}$ E. J. Son, ${ }^{124}$

J. A. Sonnenberg, ${ }^{20}$ B. Sorazu, ${ }^{43}$ F. Sorrentino, ${ }^{55}$ T. Souradeep, ${ }^{18}$ A. P. Spencer, ${ }^{43}$ A. K. Srivastava, ${ }^{100}$ A. Staley, ${ }^{46}$ M. Steinke, ${ }^{9}$ J. Steinlechner, ${ }^{43,31}$ S. Steinlechner, ${ }^{31}$ D. Steinmeyer, ${ }^{9,21}$ B. C. Stephens, ${ }^{20}$ S. P. Stevenson, ${ }^{53}$ R. Stone, ${ }^{98}$

K. A. Strain, ${ }^{43}$ G. Stratta, ${ }^{65,66}$ S. E. Strigin, ${ }^{57}$ R. Sturani, ${ }^{143}$ A. L. Stuver, ${ }^{6}$ T. Z. Summerscales, ${ }^{144}$ L. Sun, ${ }^{132}$ S. Sunil, ${ }^{100}$

P. J. Sutton, ${ }^{96}$ B. L. Swinkels ${ }^{28}$ M. J. Szczepańczyk, ${ }^{33}$ M. Tacca,${ }^{35}$ D. Talukder,${ }^{67}$ D. B. Tanner, ${ }^{5}$ M. Tápai, ${ }^{111}$

A. Taracchini, ${ }^{34}$ J. A. Taylor, ${ }^{129}$ R. Taylor, ${ }^{1}$ T. Theeg, ${ }^{9}$ E. G. Thomas, ${ }^{53}$ M. Thomas, ${ }^{6}$ P. Thomas, ${ }^{44}$ K. A. Thorne, ${ }^{6}$ K. S. Thorne, ${ }^{59}$ E. Thrane, ${ }^{127}$ S. Tiwari, ${ }^{16,90}$ V. Tiwari,${ }^{96}$ K. V. Tokmakov, ${ }^{118}$ K. Toland,${ }^{43}$ M. Tonelli, ${ }^{22,23}$ Z. Tornasi, ${ }^{43}$ C. I. Torrie, ${ }^{1}$ D. Töyrä, ${ }^{53}$ F. Travasso, ${ }^{28,40}$ G. Traylor,${ }^{6}$ D. Trifirò,,${ }^{10}$ J. Trinastic,${ }^{5}$ M. C. Tringali, ${ }^{104,90}$ L. Trozzo, ${ }^{145,23}$ K. W. Tsang, ${ }^{13}$ M. Tse,${ }^{14}$ R. Tso, ${ }^{1}$ D. Tuyenbayev,${ }^{98}$ K. Ueno, ${ }^{20}$ D. Ugolini, ${ }^{146}$ C. S. Unnikrishnan, ${ }^{112}$ A. L. Urban, ${ }^{1}$ S. A. Usman, ${ }^{96}$ K. Vahi, ${ }^{102}$ H. Vahlbruch, ${ }^{21}$ G. Vajente, ${ }^{1}$ G. Valdes,${ }^{98}$ M. Vallisneri, ${ }^{59}$ N. van Bakel,${ }^{13}$ M. van Beuzekom, ${ }^{13}$ J. F. J. van den Brand, ${ }^{71,13}$ C. Van Den Broeck, ${ }^{13}$ D. C. Vander-Hyde, ${ }^{41}$ L. van der Schaaf, ${ }^{13}$ J. V. van Heijningen, ${ }^{13}$ A. A. van Veggel, ${ }^{43}$ M. Vardaro, ${ }^{49,50}$ V. Varma, ${ }^{59}$ S. Vass, ${ }^{1}$ M. Vasúth, ${ }^{45}$ A. Vecchio,${ }^{53}$ G. Vedovato, ${ }^{50}$ J. Veitch, ${ }^{53}$ P. J. Veitch ${ }^{78}$ K. Venkateswara, ${ }^{140}$ G. Venugopalan, ${ }^{1}$ D. Verkindt, ${ }^{7}$ F. Vetrano, ${ }^{65,66}$ A. Viceré,${ }^{65,66}$ A. D. Viets, ${ }^{20}$ S. Vinciguerra, ${ }^{53}$ D. J. Vine ${ }^{58}$ J.-Y. Vinet, ${ }^{62}$ S. Vitale, ${ }^{14}$ T. Vo, ${ }^{41}$ H. Vocca,${ }^{39,40}$ C. Vorvick, ${ }^{44}$ D. V. Voss, ${ }^{5}$ W. D. Vousden,${ }^{53}$ S. P. Vyatchanin, ${ }^{57}$ A. R. Wade, ${ }^{1}$ L. E. Wade, ${ }^{81}$ M. Wade ${ }^{81}$ R. M. Wald ${ }^{86}$ R. Walet, ${ }^{13}$ M. Walker, ${ }^{2}$ L. Wallace, ${ }^{1}$ S. Walsh,${ }^{20}$ G. Wang, ${ }^{16,66}$ H. Wang, ${ }^{53}$ J. Z. Wang, ${ }^{82}$ M. Wang, ${ }^{53}$ Y.-F. Wang, ${ }^{88}$ Y. Wang, ${ }^{60}$ R. L. Ward, ${ }^{24}$ J. Warner, ${ }^{44}$ M. Was, ${ }^{7}$ J. Watchi, ${ }^{92}$ B. Weaver, ${ }^{44}$ L.-W. Wei, ${ }^{9,21}$ M. Weinert, ${ }^{9}$ A. J. Weinstein, ${ }^{1}$ R. Weiss, ${ }^{14}$ L. Wen, ${ }^{60}$ E. K. Wessel, ${ }^{11}$ P. Weßels, ${ }^{9}$ T. Westphal, ${ }^{9}$ K. Wette, ${ }^{9}$ J. T. Whelan, ${ }^{116}$ B. F. Whiting, ${ }^{5}$ C. Whittle, ${ }^{127}$ D. Williams, ${ }^{43}$ R. D. Williams, ${ }^{1}$ A. R. Williamson, ${ }^{116}$ J. L. Willis, ${ }^{147}$ B. Willke, ${ }^{21,9}$ M. H. Wimmer, ${ }^{9,21}$ W. Winkler, ${ }^{9}$ C. C. Wipf,${ }^{1}$ H. Wittel,${ }^{9,21}$ G. Woan, ${ }^{43}$ J. Woehler, ${ }^{9}$ J. Wofford, ${ }^{116}$ K. W. K. Wong, ${ }^{88}$ J. Worden,,${ }^{44}$ J. L. Wright, ${ }^{43}$ D. S. Wu, ${ }^{9}$ G. Wu, ${ }^{6}$ W. Yam, ${ }^{14}$ H. Yamamoto, ${ }^{1}$ C. C. Yancey ${ }^{72}$ M. J. Yap, ${ }^{24}$ Hang Yu, ${ }^{14}$ Haocun Yu, ${ }^{14}$ M. Yvert, ${ }^{7}$ A. Zadrożny, ${ }^{125}$ M. Zanolin, ${ }^{33}$ T. Zelenova, ${ }^{28}$ J.-P. Zendri, ${ }^{50}$ M. Zevin, ${ }^{95}$ L. Zhang, ${ }^{1}$ M. Zhang, ${ }^{133}$ T. Zhang, ${ }^{43}$ Y.-H. Zhang, ${ }^{116}$ C. Zhao, ${ }^{60}$ M. Zhou, ${ }^{95}$ Z. Zhou, ${ }^{95}$ X. J. Zhu, ${ }^{60}$ A. Zimmerman, ${ }^{85}$ M. E. Zucker, ${ }^{1,14}$ and J. Zweizig ${ }^{1}$

(LIGO Scientific and Virgo Collaboration)

${ }^{1}$ LIGO, California Institute of Technology, Pasadena, California 91125, USA

${ }^{2}$ Louisiana State University, Baton Rouge, Louisiana 70803, USA

${ }^{3}$ Università di Salerno, Fisciano, I-84084 Salerno, Italy

${ }^{4}$ INFN, Sezione di Napoli, Complesso Universitario di Monte S. Angelo, I-80126 Napoli, Italy

${ }^{5}$ University of Florida, Gainesville, Florida 32611, USA

${ }^{6}$ LIGO Livingston Observatory, Livingston, Louisiana 70754, USA

${ }^{7}$ Laboratoire d'Annecy-le-Vieux de Physique des Particules (LAPP), Université Savoie Mont Blanc, CNRS/IN2P3, F-74941 Annecy, France

${ }^{8}$ University of Sannio at Benevento, I-82100 Benevento, Italy and INFN, Sezione di Napoli, I-80100 Napoli, Italy

${ }^{9}$ Albert-Einstein-Institut, Max-Planck-Institut für Gravitationsphysik, D-30167 Hannover, Germany

${ }^{10}$ The University of Mississippi, University, Mississippi 38677, USA

${ }^{11}$ NCSA, University of Illinois at Urbana-Champaign, Urbana, IL 61801, USA

${ }^{12}$ University of Cambridge, Cambridge CB2 1TN, United Kingdom

${ }^{13}$ Nikhef, Science Park, 1098 XG Amsterdam, Netherlands 
${ }^{14}$ LIGO, Massachusetts Institute of Technology, Cambridge, Massachusetts 02139, USA

${ }^{15}$ Instituto Nacional de Pesquisas Espaciais, 12227-010 São José dos Campos, São Paulo, Brazil

${ }^{16}$ Gran Sasso Science Institute (GSSI), I-67100 L'Aquila, Italy

${ }^{17}$ INFN, Sezione di Roma Tor Vergata, I-00133 Roma, Italy

${ }^{18}$ Inter-University Centre for Astronomy and Astrophysics, Pune 411007, India

${ }^{19}$ International Centre for Theoretical Sciences, Tata Institute of Fundamental Research, Bengaluru 560089, India

${ }^{20}$ University of Wisconsin-Milwaukee, Milwaukee, Wisconsin 53201, USA

${ }^{21}$ Leibniz, Universität Hannover, D-30167 Hannover, Germany

${ }^{22}$ Università di Pisa, I-56127 Pisa, Italy

${ }^{23}$ INFN, Sezione di Pisa, I-56127 Pisa, Italy

${ }^{24}$ OzGrav, Australian National University, Canberra, Australian Capital Territory 0200, Australia

${ }^{25}$ Laboratoire des Matériaux Avancés (LMA), CNRS/IN2P3, F-69622 Villeurbanne, France

${ }^{26}$ LAL, Univ. Paris-Sud, CNRS/IN2P3, Université Paris-Saclay, F-91898 Orsay, France

${ }^{27}$ California State University Fullerton, Fullerton, California 92831, USA

${ }^{28}$ European Gravitational Observatory (EGO), I-56021 Cascina, Pisa, Italy

${ }^{29}$ Chennai Mathematical Institute, Chennai 603103, India

${ }^{30}$ Università di Roma Tor Vergata, I-00133 Roma, Italy

${ }^{31}$ Universität Hamburg, D-22761 Hamburg, Germany

${ }^{32}$ INFN, Sezione di Roma, I-00185 Roma, Italy

${ }^{33}$ Embry-Riddle Aeronautical University, Prescott, Arizona 86301, USA

${ }^{34}$ Albert-Einstein-Institut, Max-Planck-Institut für Gravitationsphysik, D-14476 Potsdam-Golm, Germany

${ }^{35}$ APC, AstroParticule et Cosmologie, Université Paris Diderot, CNRS/IN2P3, CEA/Irfu, Observatoire de Paris, Sorbonne Paris Cité, F-75205 Paris Cedex 13, France

${ }^{36}$ Korea Institute of Science and Technology Information, Daejeon 34141, Korea

${ }^{37}$ West Virginia University, Morgantown, West Virginia 26506, USA

${ }^{38}$ Center for Gravitational Waves and Cosmology, West Virginia University, Morgantown, West Virginia 26505, USA

${ }^{39}$ Università di Perugia, I-06123 Perugia, Italy

${ }^{40}$ INFN, Sezione di Perugia, I-06123 Perugia, Italy

${ }^{41}$ Syracuse University, Syracuse, New York 13244, USA

${ }^{42}$ University of Minnesota, Minneapolis, Minnesota 55455, USA

${ }^{43}$ SUPA, University of Glasgow, Glasgow G12 8QQ, United Kingdom

${ }^{44}$ LIGO Hanford Observatory, Richland, Washington 99352, USA

${ }^{45}$ Wigner RCP, RMKI, H-1121 Budapest, Konkoly Thege Miklós út 29-33, Hungary

${ }^{46}$ Columbia University, New York, New York 10027, USA

${ }^{47}$ Stanford University, Stanford, California 94305, USA

${ }^{48}$ Università di Camerino, Dipartimento di Fisica, I-62032 Camerino, Italy

${ }^{49}$ Università di Padova, Dipartimento di Fisica e Astronomia, I-35131 Padova, Italy

${ }^{50}$ INFN, Sezione di Padova, I-35131 Padova, Italy

${ }^{51}$ MTA Ë̈tvös University, "Lendulet” Astrophysics Research Group, Budapest 1117, Hungary

${ }^{52}$ Nicolaus Copernicus Astronomical Center, Polish Academy of Sciences, 00-716, Warsaw, Poland

${ }^{53}$ University of Birmingham, Birmingham B15 2TT, United Kingdom

${ }^{54}$ Università degli Studi di Genova, I-16146 Genova, Italy

${ }^{55}$ INFN, Sezione di Genova, I-16146 Genova, Italy

${ }^{56}$ RRCAT, Indore MP 452013, India

${ }^{57}$ Faculty of Physics, Lomonosov Moscow State University, Moscow 119991, Russia

${ }^{58}$ SUPA, University of the West of Scotland, Paisley PA1 2BE, United Kingdom

${ }^{59}$ Caltech CaRT, Pasadena, California 91125, USA

${ }^{60}$ OzGrav, University of Western Australia, Crawley, Western Australia 6009, Australia

${ }^{61}$ Department of Astrophysics/IMAPP, Radboud University Nijmegen, P.O. Box 9010, 6500 GL Nijmegen, Netherlands

${ }^{62}$ Artemis, Université Côte d'Azur, Observatoire Côte d'Azur, CNRS, CS 34229, F-06304 Nice Cedex 4, France

${ }^{63}$ Institut de Physique de Rennes, CNRS, Université de Rennes 1, F-35042 Rennes, France

${ }^{64}$ Washington State University, Pullman, Washington 99164, USA

${ }^{65}$ Università degli Studi di Urbino "Carlo Bo," I-61029 Urbino, Italy

${ }^{66}$ INFN, Sezione di Firenze, I-50019 Sesto Fiorentino, Firenze, Italy

${ }^{67}$ University of Oregon, Eugene, Oregon 97403, USA

${ }^{68}$ Laboratoire Kastler Brossel, UPMC-Sorbonne Universités, CNRS, ENS-PSL Research University, Collège de France, F-75005 Paris, France

${ }^{69}$ Carleton College, Northfield, Minnesota 55057, USA

${ }^{70}$ Astronomical Observatory Warsaw University, 00-478 Warsaw, Poland

${ }^{71} \mathrm{VU}$ University Amsterdam, $1081 \mathrm{HV}$ Amsterdam, Netherlands 


\footnotetext{
${ }^{72}$ University of Maryland, College Park, Maryland 20742, USA
}

${ }^{73}$ Center for Relativistic Astrophysics and School of Physics, Georgia Institute of Technology, Atlanta, Georgia 30332, USA

${ }^{74}$ Université Claude Bernard Lyon 1, F-69622 Villeurbanne, France

${ }^{75}$ Università di Napoli “Federico II,” Complesso Universitario di Monte S. Angelo, I-80126 Napoli, Italy

${ }^{76}$ NASA Goddard Space Flight Center, Greenbelt, Maryland 20771, USA

${ }^{77}$ RESCEU, University of Tokyo, Tokyo, 113-0033, Japan

${ }^{78} \mathrm{OzGrav}$, University of Adelaide, Adelaide, South Australia 5005, Australia

${ }^{79}$ Tsinghua University, Beijing 100084, China

${ }^{80}$ Texas Tech University, Lubbock, Texas 79409, USA

${ }^{81}$ Kenyon College, Gambier, Ohio 43022, USA

${ }^{82}$ The Pennsylvania State University, University Park, Pennsylvania 16802, USA

${ }^{83}$ National Tsing Hua University, Hsinchu City, 30013 Taiwan, Republic of China

${ }^{84}$ Charles Sturt University, Wagga Wagga, New South Wales 2678, Australia

${ }^{85}$ Canadian Institute for Theoretical Astrophysics, University of Toronto, Toronto, Ontario M5S 3H8, Canada

${ }^{86}$ University of Chicago, Chicago, Illinois 60637, USA

${ }^{87}$ Pusan National University, Busan 46241, Korea

${ }^{88}$ The Chinese University of Hong Kong, Shatin, NT, Hong Kong

${ }^{89}$ INAF, Osservatorio Astronomico di Padova, Vicolo dell'Osservatorio 5, I-35122 Padova, Italy

${ }^{90}$ INFN, Trento Institute for Fundamental Physics and Applications, I-38123 Povo, Trento, Italy

${ }^{91}$ Università di Roma “La Sapienza," I-00185 Roma, Italy

${ }^{92}$ Université Libre de Bruxelles, Brussels 1050, Belgium

${ }^{93}$ Sonoma State University, Rohnert Park, California 94928, USA

${ }^{94}$ Montana State University, Bozeman, Montana 59717, USA

${ }^{95}$ Center for Interdisciplinary Exploration \& Research in Astrophysics (CIERA), Northwestern University, Evanston, Illinois 60208, USA

${ }^{96}$ Cardiff University, Cardiff CF24 3AA, United Kingdom

${ }^{97}$ Universitat de les Illes Balears, IAC3-IEEC, E-07122 Palma de Mallorca, Spain

${ }^{98}$ The University of Texas Rio Grande Valley, Brownsville, Texas 78520, USA

${ }^{99}$ Bellevue College, Bellevue, Washington 98007, USA

${ }^{100}$ Institute for Plasma Research, Bhat, Gandhinagar 382428, India

${ }^{101}$ The University of Sheffield, Sheffield S10 2TN, United Kingdom

${ }^{102}$ University of Southern California Information Sciences Institute, Marina Del Rey, California 90292, USA

${ }^{103}$ California State University, Los Angeles, 5151 State University Drive, Los Angeles, California 90032, USA

${ }^{104}$ Università di Trento, Dipartimento di Fisica, I-38123 Povo, Trento, Italy

${ }^{105}$ Montclair State University, Montclair, New Jersey 07043, USA

${ }^{106}$ National Astronomical Observatory of Japan, 2-21-1 Osawa, Mitaka, Tokyo 181-8588, Japan

${ }^{107}$ Whitman College, 345 Boyer Avenue, Walla Walla, Washington 99362 USA

${ }^{108}$ School of Mathematics, University of Edinburgh, Edinburgh EH9 3FD, United Kingdom

${ }^{109}$ University and Institute of Advanced Research, Gandhinagar Gujarat 382007, India

${ }^{110}$ IISER-TVM, CET Campus, Trivandrum Kerala 695016, India

${ }^{111}$ University of Szeged, Dóm tér 9, Szeged 6720, Hungary

${ }^{112}$ Tata Institute of Fundamental Research, Mumbai 400005, India

${ }^{113}$ INAF, Osservatorio Astronomico di Capodimonte, I-80131, Napoli, Italy

${ }^{114}$ University of Michigan, Ann Arbor, Michigan 48109, USA

${ }^{115}$ American University, Washington, D.C. 20016, USA

${ }^{116}$ Rochester Institute of Technology, Rochester, New York 14623, USA

${ }^{117}$ University of Biatystok, 15-424 Biatystok, Poland

${ }^{118}$ SUPA, University of Strathclyde, Glasgow G1 1XQ, United Kingdom

${ }^{119}$ University of Southampton, Southampton SO17 1BJ, United Kingdom

${ }^{120}$ University of Washington Bothell, 18115 Campus Way NE, Bothell, Washington 98011, USA

${ }^{121}$ Institute of Applied Physics, Nizhny Novgorod, 603950, Russia

${ }^{122}$ Seoul National University, Seoul 08826, Korea

${ }^{123}$ Inje University Gimhae, South Gyeongsang 50834, Korea

${ }^{124}$ National Institute for Mathematical Sciences, Daejeon 34047, Korea

${ }^{125}$ NCBJ, 05-400 Świerk-Otwock, Poland

${ }^{126}$ Institute of Mathematics, Polish Academy of Sciences, 00656 Warsaw, Poland

${ }^{127}$ OzGrav, School of Physics \& Astronomy, Monash University, Clayton 3800, Victoria, Australia

${ }^{128}$ Hanyang University, Seoul 04763, Korea

${ }^{129}$ NASA Marshall Space Flight Center, Huntsville, Alabama 35811, USA

${ }^{130}$ ESPCI, CNRS, F-75005 Paris, France 
${ }^{131}$ Southern University and A\&M College, Baton Rouge, Louisiana 70813, USA

${ }^{132}$ OzGrav, University of Melbourne, Parkville, Victoria 3010, Australia

${ }^{133}$ College of William and Mary, Williamsburg, Virginia 23187, USA

${ }^{134}$ Indian Institute of Technology Madras, Chennai 600036, India

${ }^{135}$ IISER-Kolkata, Mohanpur, West Bengal 741252, India

${ }^{136}$ Scuola Normale Superiore, Piazza dei Cavalieri 7, I-56126 Pisa, Italy

${ }^{137}$ Université de Lyon, F-69361 Lyon, France

${ }^{138}$ Hobart and William Smith Colleges, Geneva, New York 14456, USA

${ }^{139}$ Janusz Gil Institute of Astronomy, University of Zielona Góra, 65-265 Zielona Góra, Poland

${ }^{140}$ University of Washington, Seattle, Washington 98195, USA

${ }^{141}$ King's College London, University of London, London WC2R 2LS, United Kingdom

${ }^{142}$ Indian Institute of Technology, Gandhinagar Ahmedabad Gujarat 382424, India

${ }^{143}$ International Institute of Physics, Universidade Federal do Rio Grande do Norte, Natal RN 59078-970, Brazil

${ }^{144}$ Andrews University, Berrien Springs, Michigan 49104, USA

${ }^{145}$ Università di Siena, I-53100 Siena, Italy

${ }^{146}$ Trinity University, San Antonio, Texas 78212, USA

${ }^{147}$ Abilene Christian University, Abilene, Texas 79699, USA

*Deceased, March 2016.

${ }^{\dagger}$ Deceased, March 2017.

${ }^{\ddagger}$ Deceased, February 2017.

${ }^{\S}$ Deceased, December 2016. 